\title{
ACENTOS TONALES Y VARIANTES: DECLARATIVAS EN HABLA FORMAL Y ESPONTÁNEA DE LA GOMERA (ISLAS CANARIAS)
}

\author{
Chaxiraxi Díaz Cabrera \\ Josefa Dorta Luis \\ Universidad de La Laguna \\ Laboratorio de Fonética \\ chadiaz@edu.ull.es jdorta@edu.ull.es
}

Recibido: $19 / 05 / 2015$

Aceptado: 08/09/2015

\begin{abstract}
Resumen
Este trabajo está realizado en el marco del proyecto Estudio comparativo de la entonación y del acento en zonas fronterizas del español (FFI2014-52716-P) vinculado a AMPER (Atlas Multimédia Prosodique de l'Espace Roman). El objetivo de esta investigación es hacer un estudio fonético-fonológico de oraciones declarativas (La Gomera, Islas Canarias) emitidas en dos estilos de habla, formal y espontáneo. Las oraciones fueron analizadas con subrutinas de Matlab (López Bobo et $a l, 2007)$ para extraer los valores de F0 en el núcleo de las sílabas. El etiquetaje fonético-fonológico sigue las directrices del sistema Sp_ToBI según la propuesta de Dorta ed. (2013). Se usó el umbral psicoacústico de 1,5 semitonos (Rietveld y Gussenhoven, 1985; Pamies Bertrán et al., 2002) para cuantificar la importancia de las variaciones tonales. Los resultados obtenidos permiten afianzar y ampliar la propuesta de etiquetaje fonológico postulada para el español en Dorta ed. (2013) en donde se diferenciaban tres invariantes monotonales y dos bitonales con sus correspondientes variantes para los acentos tonales de las zonas estudiadas.

PALABRAS CLAVE: entonación, acento, Sp_ToBI, corpus experimental, corpus espontáneo.
\end{abstract}

\begin{abstract}
This work has been carried out within the framework of the project Comparative study of intonation and accent in Spanish border areas (FFI2014-52716-P), which is associated to AMPER (Atlas Multimédia Prosodique de l'Espace Roman). The objective of this paper is undertaking a phonetic-phonological study of declarative sentences (La Gomera, Canary Islands) uttered in two different speaking styles; i.e. formal and spontaneous. Sentences were analyzed using Matlab sub-routines (López Bobo et al, 2007) to extract F0 values from the syllables' nuclei. The phonetic-phonological tagging follows the rules of the Sp_ToBI system according to the proposal by Dorta ed. (2013). A psycho-acoustic threshold of 1.5 semi-tones (Rietveld y Gussenhoven, 1985; Pamies Bertrán et al., 2002) was used to quantify the importance of tonal variations. Results obtained allow consolidating and broadening the proposal for phonologic tagging in Spanish by Dorta ed. (2013) that distinguishes three mono-tonal and two bi-tonal invariants with their corresponding variants for tonal accents in the study areas considered.
\end{abstract}

KEY WORDS: intonation, stress, Sp_ToBI, experimental corpus, spontaneous corpus.

\section{Introducción}

El sistema ToBI (Tones and Break Indices) en el marco del modelo Métrico-Autosegmental (Pierrehumbert, 1980) es aplicado por primera vez al español bajo la denominación de Spanish ToBI (Sp_ToBI) con la propuesta de transcripción entonativa de Beckman et al. (2002). A 
partir de entonces se sucedieron diferentes revisiones del sistema, como la de Hualde (2003), Fernández Planas y Martínez Celdrán (2003) o Pierrehumbert (2007). Entre las más recientes destacamos las de Estebas y Prieto (2008), Prieto y Roseano (2010) y Dorta (ed. 2013).

La propuesta de Estebas y Prieto (2008) fue aplicada durante años en diversos trabajos. Dicha propuesta se basó en investigaciones tradicionales sobre la entonación del español, en una revisión de artículos anteriores sobre el Sp_ToBI y en un análisis sistemático de las variedades de español peninsular de Madrid y Sevilla y de la variedad de Ciudad de México. En ella figuran las unidades fonológicas siguientes:

1) Acentos tonales (figura 1): monotonales $\left(\mathrm{L}^{*} ; \mathrm{H}^{*}\right)$ y bitonales $\left(\mathrm{L}^{*}+\mathrm{H} ; \mathrm{L}+\mathrm{H}^{*} ; \mathrm{L}+>\mathrm{H}^{*} ; \mathrm{H}+\mathrm{L}^{*}\right)$. Los tonos altos $(\mathrm{H})$ pueden presentar escalonamiento ascendente $(\mathrm{i})$ o descendente $($ !).

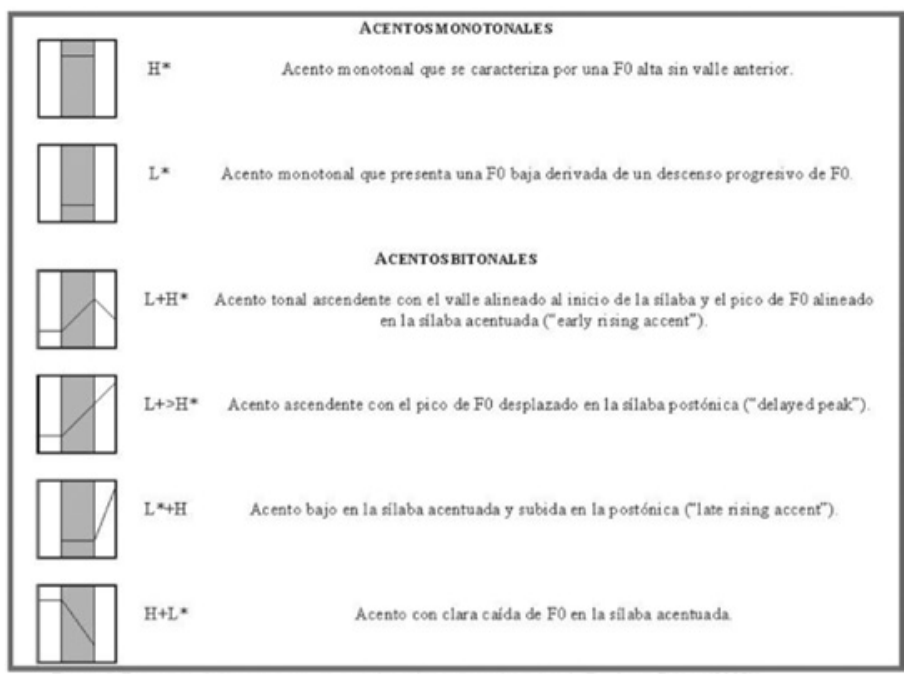

Figura 1. Esquemas de los acentos monotonales y bitonales adaptados de Estebas y Prieto (2008).

2) Tonos de frontera (figura 2): monotonales (L\%; H\%; M\%), bitonales (LH\%; HL\%; HH\%) y tritonales (LHL\%).

Posteriormente, en el 4th Sp_ToBI Workshop, celebrado en el marco del "Phonetics and Phonology in Iberia 2009”, se demostró la eficacia de las versiones revisadas del sistema ${ }^{1}$. Así, con el objetivo de presentar descripciones de una amplia variedad de enunciados en distintos dialectos del español (de España y América) con la misma metodología, se llegó a la conclusión de que existían rasgos compartidos de la lengua pero, asimismo, variación dialectal. Prieto y Roseano (2010) recogen los siguientes acentos tonales (figura 3) y tonos de frontera final (figura 4).

1 Los criterios de anotación pueden consultarse en el curso online Sp-ToBI Training Materials, en la dirección electrónica: http://prosodia.upf.edu/sp_tobi/en/ 


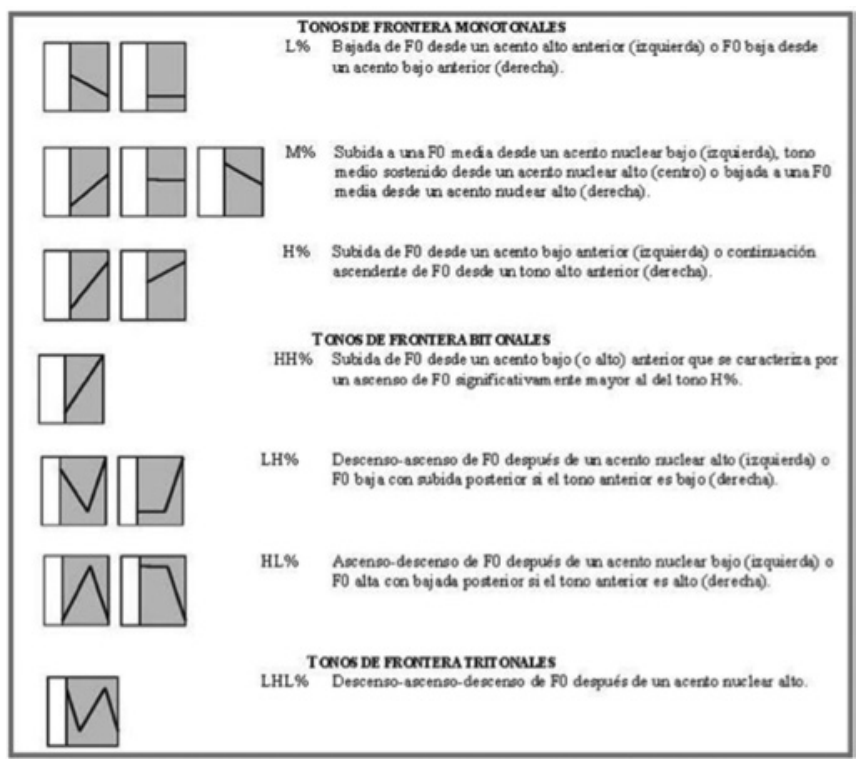

Figura 2. Esquemas de los movimientos tonales de frontera adaptados de Estebas y Prieto (2008).

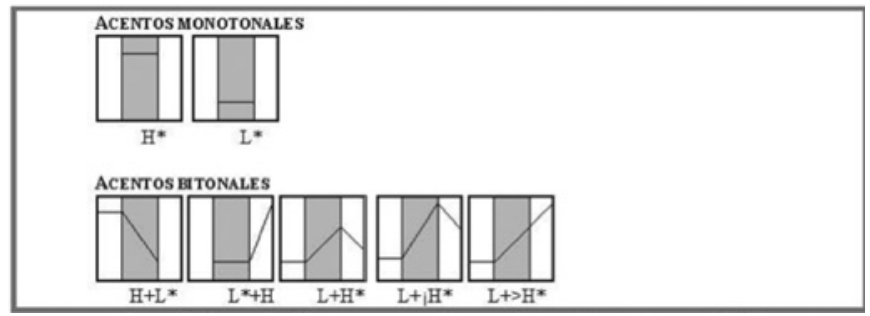

Figura 3. Adaptación de los acentos tonales recogidos en Prieto y Roseano (2010)

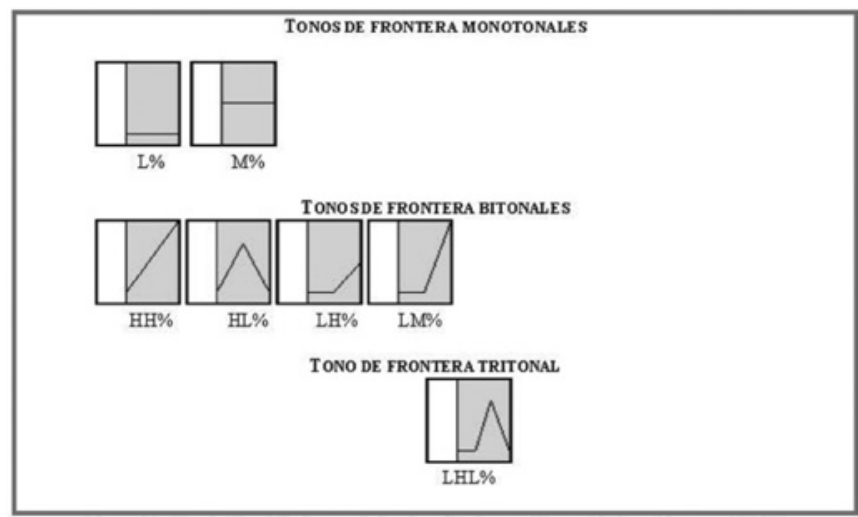

Figura 4. Adaptación de los tonos de frontera final recogidos en Prieto y Roseano (2010) 
Más recientemente, en Dorta (ed. 2013) surge una nueva propuesta de etiquetaje como resultado de la discrepancia que existía hasta entonces entre los distintos autores en los aspectos metodológicos. Esta propuesta se presenta como provisional en tanto que se deriva del análisis fonético de un corpus experimental procedente de mujeres canarias, cubanas y venezolanas. En ella se diferencian tres invariantes monotonales y dos bitonales con sus correspondientes variantes para los acentos tonales ${ }^{2}$ de las zonas canarias, venezolanas y cubanas estudiadas (figura 5) ${ }^{3}$. Nuestra pretensión, como se indica en el apartado 2, es revisar esta última propuesta a partir de análisis más exhaustivos, tanto de corpus experimental como espontáneo (véase en el apartado 3.4. "El etiquetaje prosódico" nuestro posicionamiento al respecto).

\begin{tabular}{|c|c|c|}
\hline ESTRUCTURARKONUNDA & ESTRUCTURA SUTEREICLAL & NIVELACOSTICO \\
\hline Invariante fonologica & Variantes & Esquema del contorno tonal \\
\hline \multirow{2}{*}{$/ \mathbf{L} * \mathbf{H} /$} & {$\left[L^{*++11]}\right.$} & \\
\hline & {$\left[\mathrm{L}^{*+1}+1 \mathrm{H}\right]$} & \\
\hline \multirow{2}{*}{$/ \mathbf{L}+\mathbf{H}^{*} /$} & {$\left[\mathrm{I}+\mathrm{H}^{*}\right]$} & \\
\hline & {$\left[\mathrm{L}+\mathrm{S} \mathrm{H}^{*}\right]$} & \\
\hline \multirow{2}{*}{$/ \mathbf{1} *$} & {$[L *]$} & \\
\hline & {$[: \mathbf{H}+\mathbf{L}+]$} & \\
\hline \multirow{5}{*}{$/ \mathbf{H}^{*} /$} & {$\left[\mathrm{H}^{*}\right]$} & \\
\hline & {$\left[: \mathrm{H}^{*}\right]$} & \\
\hline & {$\left[: \mathbf{H}^{*}\right]$} & \\
\hline & {$\left[\mathrm{L}+\mathrm{II}^{*}\right]$} & \\
\hline & {$\left[\mathrm{L}+; \mathrm{H}^{*}\right]$} & \\
\hline ! $\mathrm{H}^{*}$ & {$\left[\mathbf{L}+\left[\mathbf{H}^{*}\right]\right.$} & \\
\hline
\end{tabular}

Figura 5. Representación de la estructura profunda, superficial y esquema acústico de los acentos tonales (Dorta ed., 2013)

2 Solo en una zona de las tres que consideran en Venezuela (Mérida) se da la particularidad de que la oposición $/ ! \mathrm{H}^{*} /$ vs. $/ \mathrm{H}^{*} /$ es funcional. Por tanto, si no se diera esta circunstancia, solo tendríamos cuatro acentos tonales invariantes, dos monotonales y dos bitonales.

3 La pretensión de los autores es revisar la propuesta a partir de análisis más exhaustivos, tanto de corpus experimental como espontáneo. 
Asimismo, el tono de frontera final tiene tonos invariantes y sus correspondientes variantes (figura 6).

\begin{tabular}{|c|c|c|}
\hline ESTRUCTURA PROFUNDA & ESTRUCTURA SUPERFICIAL & NIVEL ACÚSTICO \\
\hline Invariante fonológica & Variantes & Esquema del contorno tonal \\
\hline \multirow{2}{*}{$/ \mathrm{H} \%$} & {$[\mathrm{H} \%]$} & $\mathrm{TM}$ \\
\hline & {$[\mathrm{MH} \%]$} & TM \\
\hline \multirow{3}{*}{ /L\%/ } & [L\%] & $\mathrm{MM}$ \\
\hline & {$[\mathrm{ML} \%]$} & $-\mathrm{TM}$ \\
\hline & {$\left[\mathrm{H}_{\mathrm{L}} \%\right]$} & тM \\
\hline
\end{tabular}

Figura 6. Representación de la estructura profunda, superficial y esquema acústico de los tonos de frontera (Dorta ed., 2013)

\section{Objetivo}

El objetivo de este trabajo es realizar un estudio fonético-fonológico de la modalidad oracional declarativa del habla de la isla de La Gomera en el marco del proyecto Estudio comparativo de la entonación y del acento en zonas fronterizas del español (FFI201452716-P) vinculado a AMPER (Atlas Multimédia Prosodique de l'Espace Roman). Pretendemos revisar y aplicar los supuestos teóricos esbozados en Dorta (ed. 2013) ampliando el corpus experimental a las oraciones con expansión en los sintagmas de frontera añadiendo, además, un corpus de tipo espontáneo. De este modo, nos proponemos determinar las estructuras tonales del nivel fonológico (invariantes) a partir de las estructuras superficiales (variantes).

\section{Metodología}

\subsection{Informantes}

Los informantes seleccionados para este trabajo son seis con edades comprendidas entre los 18 y los 55 años, con estudios y sin estudios superiores, procedentes de zonas urbanas y rurales de la isla colombina. Véase la tabla número $1^{4}$.

4 En la tabla, + corresponde a estudios superiores; - equivale a sin estudios superiores. 


\begin{tabular}{|c|c|c|c|}
\hline \multicolumn{4}{|c|}{ INFORMANTES } \\
\hline \multicolumn{2}{|c|}{ Zona urbana: San Sebastián } & \multicolumn{2}{c|}{ Zona Rural: Valle Gran Rey } \\
\hline SEXo & NiVEL DE ESTUDIOs & SEXo & NIVEL DE ESTUDIOS \\
\hline Mujer & - & Mujer & - \\
\hline Hombre & - & Hombre & - \\
\hline Mujer & + & & \\
\hline Hombre & + & & \\
\hline
\end{tabular}

Tabla 1

\subsection{Corpus}

\subsubsection{Corpus experimental}

Está constituido por dos conjuntos de oraciones emitidas mediante elicitación textual sin matices emocionales: el primero lo integran oraciones SVO de 11 sílabas del tipo $S N$ (sintagma nominal) $+S V$ (sintagma verbal) + SPrep (sintagma preposicional) sin expansión en los sintagmas nominal o preposicional como por ejemplo: el saxofón se toca con obsesión; el otro conjunto consta de oraciones de 14 y de 15 sílabas con expansión en los sintagmas de frontera. En el sintagma nominal y en el preposicional el núcleo lo forma una palabra trisílaba de diferente tipología acentual. Todas estas combinaciones dan como resultado 63 oraciones declarativas, lo que supone 378 secuencias teniendo en cuenta el conjunto de los seis informantes considerados en este estudio; al haberse realizado tres repeticiones de cada oración el corpus implica 1134 oraciones. La importancia de este tipo de corpus radica en que será el único que estará representado en la base de datos de AMPER internacional con sede en Grenoble (Francia) ya que permite comparar mejor la prosodia de todas las variedades y lenguas románicas al estar constituido por idénticas estructuras en todas ellas. En la tabla 2 se presenta un resumen.

\begin{tabular}{|c|c|c|c|c|}
\hline \multicolumn{7}{|c|}{ CorPus EXPERIMENTAL } \\
\cline { 1 - 4 } Tipo de oración & 3 repeticiones & 6 informantes & \multirow{2}{*}{ Total } \\
\hline Sin expansión & 9 & 27 & 162 & \multirow{2}{*}{1134} \\
\cline { 1 - 3 } Con expansión en el sujeto & 27 & 81 & 486 & \\
\cline { 1 - 3 } Con expansión en el objeto & 27 & 81 & 486 & \\
\hline
\end{tabular}

Tabla 2

\subsubsection{Corpus espontáneo}

Por otro lado, se consideró un corpus más espontáneo de dos tipos: semiespontáneo o inducido (CS) y espontáneo o map task (CE) cuya importancia estriba en que permitirá 
contrastar los resultados obtenidos en el corpus experimental y, por tanto, ver la representatividad de estos últimos. El primero (CS) está constituido por frases que se obtienen mediante el planteamiento al informante de cuestiones de uso muy cotidiano. Por ejemplo: "Estás almorzando con unos amigos y quieres pan: ¿Cómo lo pedirías?”. La respuesta previsible puede ser: "por favor, pásame el pan"; "dame el pan”, etc. El segundo tipo de corpus (CE) se obtiene mediante un sistema de recogida de datos que se plantea a partir de mapas: el informante y el entrevistador deben salir de un punto geográfico y llegar a un destino especificado. Puesto que los dos mapas no son idénticos, ello motivará una serie de preguntas y respuestas por parte de ambos intervinientes. El corpus definitivo está formado por 71 oraciones declarativas con esquema acentual llano en su mayoría y, en menor medida, agudo; en ningún caso fue esdrújulo. Véase en la tabla 3 la distribución del corpus.

\begin{tabular}{|c|c|c|}
\hline \multicolumn{2}{|c|}{ CoRPuS ESPONTÁNEO } \\
\hline \multicolumn{2}{|c|}{ Tipo de corpus } & \multirow{2}{*}{ Total } \\
\cline { 1 - 2 } Semiespontáneo & 52 & \\
\hline Espontáneo & 19 & \\
\hline
\end{tabular}

Tabla 3

Por otra parte, para la delimitación de las oraciones declarativas consideramos el relieve tonal de la frase teniendo en cuenta el trabajo de Torres Álvarez 20005. Por ello, separamos las declarativas que tienen un solo pico tonal máximo, cuya extensión va generalmente de 1 a 7 sílabas (71,1\% en el corpus CS y un 92,8 \% en el CE), de las que presentan más de una cumbre tonal máxima y que superan dichas cifras (+7 sílabas; suponen el $100 \%$ en ambos tipos de corpus).

\subsection{Extracción y análisis acústico de los datos}

El corpus se grabó con una grabadora Marantz PMD 222 y un micrófono direccional Shure SM 48 en un entorno cómodo para el informante. La señal analógica grabada se digitalizó con el programa Goldwave (versión 4.25) convirtiendo cada una de las frases en ficheros .wav, una vez eliminados los posibles ruidos y optimizada la señal resultante que tiene una frecuencia de muestreo de $18.000 \mathrm{~Hz}$. Los ficheros .wav se etiquetaron según la codificación de AMPERCan de la manera siguiente: 9 dígitos + extensión ${ }^{6}$.

5 En el estudio sobre las declarativas del español de Canarias, Torres Álvarez (2000) corrobora que en un $86,28 \%$ las oraciones monocumbres son cortas (de 1 a 7 sílabas) y en un 92,31\% que las oraciones bicumbres son largas (+7 sílabas).

6 Los 9 dígitos de la codificación tienen el siguiente significado: W (dominio lingüístico español); C (variedad español de Canarias); o (isla de La Gomera); número impar (mujer) y número par (hombre): los números 1-2 corresponden a zona urbana sin estudios superiores; los números 3-4 se adjudican a zona rural sin estudios superiores y los números 5-6 a zona urbana con estudios superiores. Los dígitos 5, 6, 7 son letras para identificar cada oración; con el dígito 8 se representa la modalidad: a (declarativa)/i (interrogativa) y, por último, con el dígito 9 se indica el número de repetición de la frase (1, 2 ó 3). P.ej.: una oración codificada como WCo1kwka1 nos da la siguiente información: primera repetición de la oración declarativa con estructura SN A + SPrep A (sintagma nominal agudo más sintagma preposicional agudo) emitida por una mujer urbana sin estudios superiores de la 
El análisis acústico del corpus experimental se realizó con rutinas creadas ad hoc en el año 2006 por el Laboratorio de Fonética de la Universidad de Oviedo (grupo AMPERAstur) para el entorno Matlab (versión 7.0.4.7); para el análisis del corpus semiespontáneo y espontáneo, en cambio, se empleó un script para Praat proporcionado por el Centre de Dialectologie de l'Universitè Stendhal-Grenoble III (AMPER Internacional).

\subsection{El etiquetaje prosódico}

Como todos sabemos, hay elementos que son normales en el habla de una comunidad sin que por ello pertenezcan al sistema. Esta vertiente intermedia la definía Coseriu como "norma":

La norma refleja el equilibrio del sistema en un determinado momento y, cambiando la norma, cambia ese equilibrio, hasta volcarse totalmente de un lado o de otro. [...] En latín clásico era normal la declinación desinencial, pero en muchos casos se empleaban, además, preposiciones que ya de por sí indicaban suficientemente la función: la norma se desplazó cada vez más hacia el empleo de las preposiciones, hasta que el equilibrio del sistema se volcó, quedando de la antigua declinación, en la mayoría de las lenguas romances actuales, apenas la oposición entre singular y plural (Coseriu, 1962: 107-108).

Se aleja así este autor de la dicotomía saussureana que ignora el punto en el que lengua y habla se encuentran, estableciendo un "sistema normal" distinto del "funcional" representado por la lengua. Por tanto, siguiendo a Coseriu, la norma lingüística supone un primer grado de abstracción y el sistema el segundo. A propósito, Sosa señala lo siguiente en el plano de la entonación:

Ahora bien, como en español varias de esas secuencias tonales básicas varían drásticamente de un dialecto a otro, se debe concluir que elementos con el mismo valor pueden tener distinta forma fónica de acuerdo al dialecto. En los casos en que esto ocurre, estas diferencias son tan notorias y constantes que por lo menos algunas de estas deben ser igualmente sistemáticas (Sosa, 1999: 181).

En la presente investigación intentaremos acercarnos al primer grado de abstracción, representado por la norma, con el objetivo de sistematizar la variación entonativa en la comunidad lingüística canaria de La Gomera. Nuestra propuesta de etiquetaje prosódico variantes ha sido aplicada en trabajos recientes del grupo ProFonDis (Dorta ed., 2013) y comparte algunos de los principios ya defendidos en Fernández Planas y Martínez Celdrán (2003) como, por ejemplo, que debe llegarse a las estructuras profundas o invariantes a partir de las estructuras superficiales.

isla de La Gomera. WCo1: zona urbana sin estudios superiores en voz femenina; WCo2: zona urbana sin estudios superiores en voz masculina; WCo3: zona rural sin estudios superiores en voz femenina. WCo4: zona rural sin estudios superiores en voz masculina. WCo5: zona urbana con estudios superiores en voz femenina. WCo6: zona urbana con estudios superiores en voz masculina.

7 Licencia $n^{\circ}$ 256105. Para más información sobre el programa y las subrutinas creadas por el Centre de Dialectologie de Grenoble véase Romano et al. (2005); sobre la adaptación realizada por el grupo Amper-Astur, véase López-Bobo et al. (2007). 
Hay que destacar, por otro lado, que como es usual en el campo que estudiamos, hemos considerado el umbral psicoacústico situado en 1,5 semitonos (Rietveld y Gussenhoven, 1985; Pamies Bertrán et al., 2002) con la finalidad de que las variaciones tonales puedan ser cuantificables. No obstante, a diferencia de Fernández Planas y Martínez Celdrán (2003) o Toledo (2006), por ejemplo, no nos centramos en las diferencias que se establecen entre las vocales que forman los grupos tónicos (pretónica-tónica-postónica) para etiquetar los acentos tonales de las oraciones, sino que consideramos los cambios en la trayectoria de $\mathrm{F}_{0}$, esto es, los movimientos de ascenso y descenso. Por ejemplo, etiquetamos $\left[\mathrm{L}^{*}+\mathrm{H}\right]$ en el primer acento tonal de la oración si el ascenso inicial culmina después de la tónica y el movimiento no alcanza el semitono y medio en el intervalo valle-tónica; si por el contrario alcanza el umbral perceptivo etiquetaremos $\left[\mathrm{L}+>\mathrm{H}^{*}\right]$. En lo que respecta al tono de frontera final, tomamos como referencia el tono medio del informante distinguiendo, por ello, diferentes tonos invariantes ( $\mathrm{H} \% ; \mathrm{L} \% ; \mathrm{M} \% /)$ en función de si el final supera o no la media: detallaremos la relevancia más o menos perceptiva de la relación final-media a partir de los diferentes alótonos considerados en la frontera final. Asimismo, teniendo en cuenta que en la caracterización tonal de las unidades mínimas tonales del AM se excluyen explícitamente del análisis toda referencia a elementos extratonales -como la longitud relativa de las vocales (Sosa, 1999:70)-, en nuestra caracterización fonológica no se considerará la duración vocálica (ni la intensidad) como por ejemplo sucede en Fernández Planas y Martínez Celdrán (2003). En definitiva, como se ha dicho ya, aplicaremos la propuesta que hace el grupo ProFonDis en Dorta (ed. 2013) para representar lo fonético (estructura superficial) y lo fonológico (estructura profunda).

\section{Descripción fonológica de las declarativas}

Teniendo en cuenta que el modelo fonológico en que vamos a basar nuestro estudio es el sistema ToBI (Tones and Break Indices) en el marco del modelo Métrico-Autosegmental (Pierrehumbert, 1980), en los apartados que se insertan a continuación describiremos las propuestas de etiquetaje más recientes (v.gr. Prieto y Roseano eds., 2010 o Dorta ed., 2013).

\subsection{El español general}

En Prieto y Roseano (eds. 2010) ${ }^{8}$, Estebas y Prieto (capítulo 2: 17-48), por un lado, y López-Bobo y Cuevas Alonso (capítulo 3: 49-85), por otro, describen las oraciones declarativas neutras de foco amplio en el español madrileño y cántabro, respectivamente. En las dos zonas del español peninsular las declarativas se caracterizan por un contorno $\mathrm{L}+>\mathrm{H}^{*}$ en el acento prenuclear y $\mathrm{L}^{*} \mathrm{~L} \%$ en el nuclear y tono de frontera final, es decir, el contorno entonativo está formado por un acento prenuclear ascendente, cuyo pico está desplazado a la sílaba postónica y un acento nuclear bajo en la sílaba tónica seguido de un tono de frontera bajo. Véase la figura 7 con la que ilustran Estebas y Prieto el contorno declarativo (Prieto y Roseano eds., 2010 capítulo 2: 24).

8 Los datos se pueden consultar igualmente en el Atlas Interactivo de la entonación del español: http://prosodia.upf.edu/atlasentonacion/cerca/index.php 


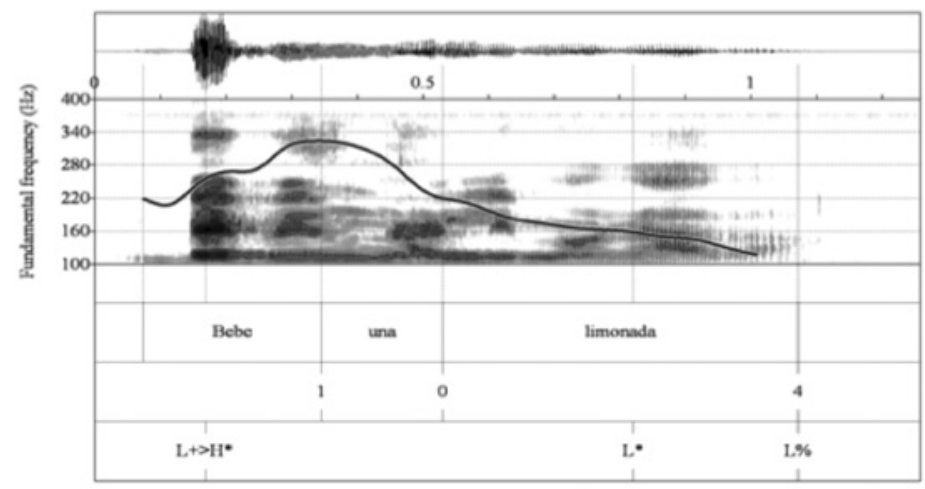

Figura 7. Contorno declarativa de foco amplio. Prieto y Roseano (eds. 2010, capítulo 2:24).

\subsection{El español hispanoamericano}

En Prieto y Roseano (eds. 2010) se describe el español andino de Mérida (en Venezuela, Astruc et al., capítulo 7: 191-226) y Quito (en Equador, O’Rourke, capítulo 8: 227-253), el argentino de Buenos Aires (Christoph et al., capítulo 10: 285-317) y el caribeño de San Juan de Puerto Rico (Armstrong, capítulo 6: 155-189) y de Santiago de los Caballeros en Santo Domingo (Willis, capítulo 5: 123-153); además se aportan datos de Santiago de Chile (Héctor Ortiz et al., capítulo 9: 255-283) y México D.F. (de la Mota et al., capítulo 11: 319350). En relación con las declarativas neutras, en la mayor parte de estos trabajos el patrón no difiere del propuesto para el español general en tanto que el primer acento es $\mathrm{L}+>\mathrm{H}^{*}$, el nuclear $\mathrm{L}^{*}$ y el tono de frontera final $\mathrm{L} \%$. No obstante, se dan algunas diferencias:

a) El primer acento en Buenos Aires es $\mathrm{H}^{*}$ y en Puerto Rico $\mathrm{L}^{*}+\mathrm{H}$ y no el desplazado $\mathrm{L}+>\mathrm{H}^{*}$.

b) El acento nuclear presenta un movimiento ascendente en Mérida (! $\left.\mathrm{H}^{*}\right)$, Santiago de los Caballeros $\left(\mathrm{L}+\mathrm{H}^{*}\right)$ y Santiago de Chile $\left(\mathrm{L}+\mathrm{H}^{*}\right)$ aunque, salvo en la variedad dominicana, desciende finalmente.

Podemos ver un ejemplo en la figura 8 correspondiente a la zona andina venezolana (Astruc et al., capítulo 7: 197).

Respecto del español de Cuba, en Dorta (ed. 2013) se etiqueta en líneas generales el último acento de la frase y el tono de frontera final acorde con el patrón esbozado para el español general, esto es, descendente ( $\mathrm{L}^{*} \mathrm{~L} \%$ ); en cambio, para el primer acento tonal de las declarativas cubanas (y venezolanas) ${ }^{9}$, se propone la invariante $/ \mathrm{L}+\mathrm{H}^{*} /$ cuya variación puede ser $\left[\mathrm{L}+>\mathrm{H}^{*}\right]$ o $\left[\mathrm{L}+\mathrm{H}^{*}\right]$, es decir, se determina un movimiento ascendente durante el primer acento que puede concluir o no en la tónica. A idénticas conclusiones se llegó en un estudio posterior en el que, aplicando la propuesta esbozada en el libro de referencia, se comparó la zona andina de Mérida con la de Medellín (Díaz et al., en prensa).

9 También para Canarias como veremos en el apartado que sigue a continuación (4.3.). 


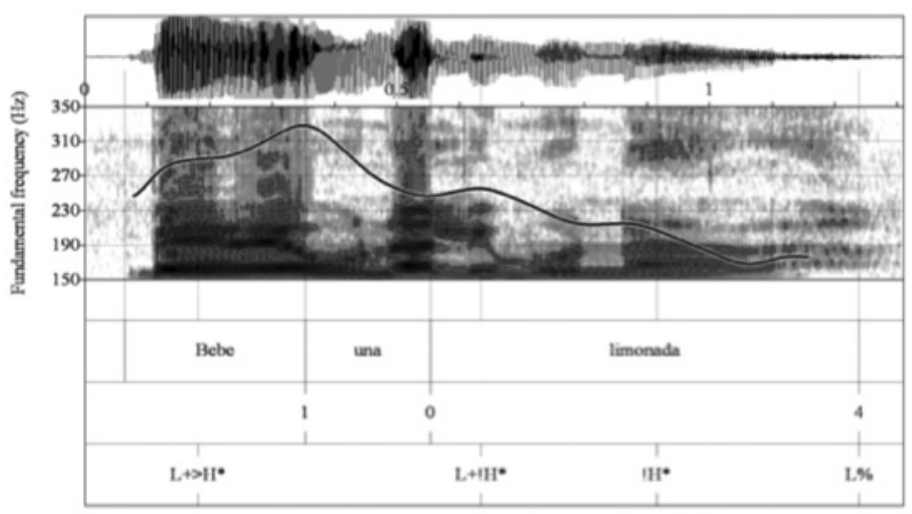

Figura 8. Contorno declarativa de foco amplio. Prieto y Roseano (eds. 2010, capítulo 7: 197).

\subsection{El español de Canarias}

En lo que se refiere a Canarias, en el estudio de Cabrera y Vizcaíno (Prieto y Roseano eds. 2010, capítulo 4: 87-121), se describen las declarativas de Las Palmas de Gran Canaria de acuerdo con los presupuestos teóricos de otros autores del libro en el que se inserta el estudio. Así, las declarativas neutras se caracterizan por un patrón tonal que no se aleja del propuesto para el español peninsular, esto es, con un ascenso en el prenúcleo que finaliza con un pico desplazado a la derecha del acento $\left(\mathrm{L}+>\mathrm{H}^{*}\right)$ y un descenso durante el núcleo que llega hasta el final absoluto (L* L\%). En la figura 9 se ilustra el contorno señalado por los autores.

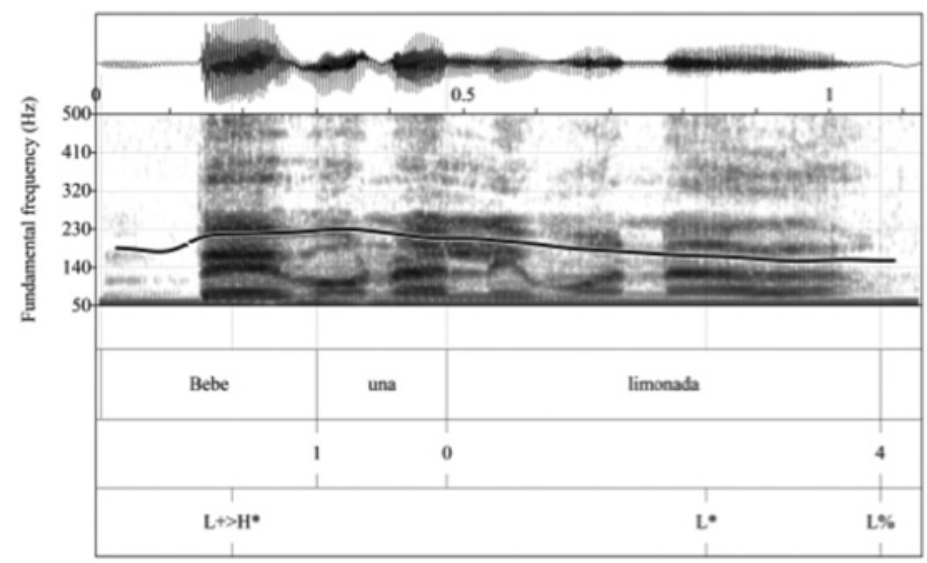

Figura 9. Contorno declarativa de foco amplio. Prieto y Roseano (eds. 2010, capítulo 4: 93).

Los estudios de tipo fonológico en el ámbito de AMPER en Canarias, si bien son más recientes, coinciden con los autores en el etiquetaje fonológico del último acento de la 
oración así como el tono de frontera final para todas las islas del archipiélago (Dorta ed., 2013), con la salvedad del primer acento tonal en el que, como apuntábamos en el apartado anterior, se concibe que la invariante es $\mathrm{L}+\mathrm{H}^{*}$ (y no $\mathrm{L}+>\mathrm{H}^{*}$ ) cuya variación puede ser $\left[\mathrm{L}+>\mathrm{H}^{*}\right]$ o $\left[\mathrm{L}+\mathrm{H}^{*}\right]$.

\section{La interpretación de los movimientos tonales en las declarativas de La Gomera}

\subsection{Corpus experimental}

\subsubsection{Sin expansión}

De acuerdo con la propuesta que aplicamos, el etiquetaje fonético de los tres acentos tonales de este tipo de oraciones y del tono de frontera final de las curvas de los seis informantes gomeros es el que mostramos en la tabla 4 .

\begin{tabular}{|c|c|c|c|c|c|c|c|c|c|c|}
\hline \multirow{3}{*}{\multicolumn{2}{|c|}{ INFORMANTES }} & \multicolumn{9}{|c|}{ Variantes } \\
\hline & & \multicolumn{3}{|c|}{ AGUDO } & \multicolumn{3}{|c|}{ LLANO } & \multicolumn{3}{|c|}{ ESDRÚJULO } \\
\hline & & SN & SV & SPrep & SN & SV & SPrep & SN & SV & SPrep \\
\hline \multirow{2}{*}{$\begin{array}{l}\text { Zona urbana } \\
\text { sin estudios }\end{array}$} & WCo1 & $\mathrm{L}+>\mathrm{H}^{*}$ & $\mathrm{~L}^{*}+\mathrm{H}$ & L*L\% & $\mathrm{L}^{*}+\mathrm{H}$ & $\mathrm{L}^{*}+\mathrm{H}$ & $\mathrm{L} * \mathrm{~L} \%$ & $\mathrm{~L}+>\mathrm{H}^{*}$ & $\mathrm{~L}^{*+\mathrm{H}}$ & $\mathrm{L} * \mathrm{~L} \%$ \\
\hline & $\mathrm{WCo} 2$ & $\mathrm{~L}+>\mathrm{H}^{*}$ & $\mathrm{~L}^{*}$ & $\mathrm{~L} * \mathrm{~L} \%$ & $\mathrm{~L}+>\mathrm{H}^{*}$ & $\mathrm{~L}^{*}$ & $\mathrm{~L} * \mathrm{~L} \%$ & $\mathrm{~L}+>\mathrm{H}^{*}$ & $\mathrm{~L}^{*}$ & $\mathrm{~L} * \mathrm{~L} \%$ \\
\hline \multirow{2}{*}{$\begin{array}{c}\text { Zona rural } \\
\text { sin estudios }\end{array}$} & WCo3 & $\mathrm{L}+>\mathrm{H}^{*}$ & $\mathrm{H}^{*}$ & $\mathrm{~L} * \mathrm{~L} \%$ & $\mathrm{~L}+>\mathrm{H}^{*}$ & $\mathrm{~L}^{*}$ & $\mathrm{~L} * \mathrm{~L} \%$ & $\mathrm{~L}+>\mathrm{H}^{*}$ & $\mathrm{~L}^{*}$ & $\mathrm{~L} * \mathrm{~L} \%$ \\
\hline & WCo4 & $\mathrm{L}+\mathrm{H}^{*}$ & $\mathrm{H}^{*}$ & L*L\% & $\mathrm{L}+>\mathrm{H}^{*}$ & $\mathrm{H}^{*}$ & $\mathrm{~L} * \mathrm{~L} \%$ & $\mathrm{~L}+>\mathrm{H}^{*}$ & $\mathrm{H}^{*}$ & $\mathrm{~L} * \mathrm{~L} \%$ \\
\hline \multirow{2}{*}{$\begin{array}{l}\text { Zona urbana } \\
\text { con estudios }\end{array}$} & WCo5 & $\mathrm{L}+\mathrm{H}^{*}$ & $\mathrm{~L}^{*}$ & $\mathrm{~L} * \mathrm{~L} \%$ & $\mathrm{~L}+>\mathrm{H}^{*}$ & $\mathrm{~L}^{*}$ & $\mathrm{~L} * \mathrm{~L} \%$ & $\mathrm{~L}+\mathrm{H}^{*}$ & $\mathrm{~L}^{*}$ & $\mathrm{~L} * \mathrm{~L} \%$ \\
\hline & WCo6 & $\mathrm{L}+\mathrm{H}^{*}$ & $\mathrm{~L}^{*}$ & $\mathrm{~L} * \mathrm{~L} \%$ & $\mathrm{~L}+>\mathrm{H}^{*}$ & $\mathrm{~L}^{*}+\mathrm{H}$ & $\mathrm{L} * \mathrm{~L} \%$ & $\mathrm{~L}+>\mathrm{H}^{*}$ & $\mathrm{~L}^{*}$ & $\mathrm{~L} * \mathrm{~L} \%$ \\
\hline
\end{tabular}

\section{Tabla 4}

En la tabla precedente puede observarse que las declarativas sin expansión están constituidas por dos acentos prenucleares y uno nuclear:

a) En el prenúcleo, determinamos como invariante del primer acento (SN) un contorno ascendente bitonal $\left(/ \mathrm{L}+\mathrm{H}^{*} /\right)$ que puede realizarse dentro de los límites de la tónica ([L+H*], $22,2 \%$ ) o con el pico desplazado a la postónica ([L+> $\left.\mathrm{H}^{*}\right], 72,2 \%$ ); el segundo acento del pretonema (SV) presenta mayor variabilidad que el anterior en tanto que encontramos dos variantes monotonales $\left(\left[\mathrm{H}^{*}\right]\right.$ y $\left.\left[\mathrm{L}^{*}\right]\right)$ y una bitonal $\left(\left[\mathrm{L}^{*}+\mathrm{H}\right]\right)$.

En lo que respecta a las variantes monotonales, observamos lo siguiente:

$\left.1^{\circ}\right)\left[\mathrm{H}^{*}\right]\left(/ \mathrm{H}^{*} /\right)$ : se reduce al ámbito rural (no siempre en todas las combinaciones [22,2\%] $v$. $g r$ WCo3) por lo que parece una característica de este tipo de habla. Recordemos que Sosa indicaba (1999:228) que la impresión de "agudo o grave" que puede connotar un dialecto guarda relación con la frecuencia de uso de tonos $\mathrm{H}^{*}$.

$\left.2^{\circ}\right)\left[\mathrm{L}^{*}\right]\left(/ \mathrm{L}^{*} /\right)$ : esta variante es la más sistemática en la configuración del segundo acento prenuclear de las declarativas puesto que cuenta con el mayor porcentaje de realización (55,5\% de los casos). 
La variante bitonal $\left[\mathrm{L}^{*+\mathrm{H}}\right]\left(/ \mathrm{L}^{*+\mathrm{H}} /\right)$, esto es, la caracterizada por un acento bajo durante la tónica seguido de un tono alto, se da en un $22,2 \%$. Nótese que se registra en las tres combinaciones de la mujer de zona urbana sin estudios (WCo1).

b) El acento nuclear de las declarativas es descendente y culmina con un tono de frontera bajo en todos los casos $(/ \mathrm{L} * \mathrm{~L} \% /)$.

En la taba 5 incluimos las invariantes y sus variantes más frecuentes con los porcentajes de aparición ${ }^{10}$.

\begin{tabular}{|c|c|c|c|c|c|c|c|}
\hline \multicolumn{2}{|c|}{ Invariantes y variantes } \\
\hline \multicolumn{2}{|c|}{ SN } & \multicolumn{2}{|c|}{ SV } & \multicolumn{4}{c|}{ SPrep } \\
\hline \multicolumn{2}{|c|}{ Acento 1 } & \multicolumn{2}{|c|}{ Acento 2 } & \multicolumn{2}{|c|}{ Acento 3 } & \multicolumn{2}{c|}{$\%$} \\
\hline $\mathbf{I}$ & $\mathbf{V}$ & $\mathbf{I}$ & $\mathbf{V}$ & $\mathbf{I}$ & $\mathbf{V}$ & $\mathbf{I}$ & $\mathbf{V}$ \\
\hline & $\mathrm{L}+\mathrm{H}^{*} 22,2 \%$ \\
$\mathbf{L}+\mathbf{H}^{*}$ & $\mathrm{~L}+>\mathrm{H}^{*} 72,2 \% 0^{11}$ & $\mathbf{L}^{*}$ & $\mathrm{~L}^{*} 55,5 \%{ }^{12}$ & $\mathbf{L}^{*}$ & $\mathrm{~L}^{*} 100 \%$ & $\mathbf{L} \%$ & $\mathrm{~L} \% 100 \%$ \\
\hline
\end{tabular}

\section{Tabla 5}

Finalmente, de acuerdo con la propuesta aplicada, ofrecemos en la tabla 6 la interpretación de los dos acentos prenucleares de las declarativas sin expansión ( $\mathrm{SN}$ y SV) y, asimismo, del acento nuclear y tono de frontera final (SPrep), esto es, las invariantes.

\begin{tabular}{|c|c|c|}
\hline \multicolumn{3}{|c|}{ Invariantes } \\
\hline SN & SV & SPrep \\
\hline $\mathrm{L}^{*} \mathrm{H}^{*}$ & $\mathrm{~L}^{*}$ & $\mathrm{~L} * \mathrm{~L} \%$ \\
\hline
\end{tabular}

\section{Tabla 6}

De lo anterior, podemos concluir que lo que caracteriza a las declarativas sin expansión de manera sistemática es un ascenso durante el primer acento tonal que deja a la tónica en un tono alto $\left(/ \mathrm{L}+\mathrm{H}^{*} /\right)$; un acento final descendente que culmina en un tono de frontera bajo $\left(/ \mathrm{L}^{*} \mathrm{~L} \% /\right) \mathrm{y}$, generalmente, la presencia de un tono bajo $(/ \mathrm{L} * /)$ en el último acento prenuclear, tal como se ilustra en las figuras 10 y 11 correspondientes a una mujer (WCo5) y a un hombre (WCo2).

10 No hay que olvidar que al tratarse de las curvas de cada informante y no de las medias del conjunto de los informantes como se ha hecho en trabajos anteriores (v.gr. Dorta, Martín y Díaz, 2015) hay mayor probabilidad de variación.

11 El 5,6\% restante corresponde al etiquetaje $\left[\mathrm{L}^{*}+\mathrm{H}\right]$ que de ninguna manera podemos considerar variante de $\left[\mathrm{L}+\mathrm{H}^{*}\right]$ sino una realización esporádica como indica el propio porcentaje.

12 El 44,4\% que falta en la casilla corresponde a la realización $\left[\mathrm{H}^{*}\right](22,2 \%)$ y [L*+H] $(22,2 \%)$. 


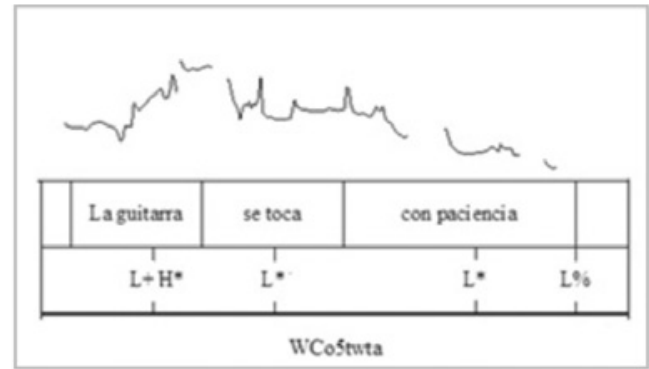

WCo5 (Sexo femenino)
Figuras 10-11

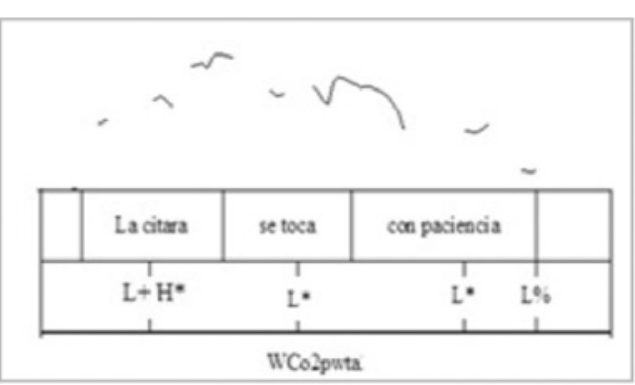

WCo3 (Sexo masculino)

\subsubsection{Con expansión en los sintagmas de frontera}

a) El sintagma sujeto consta ahora de un núcleo (N) seguido de una expansión (Exp); en ambos casos aparecen los tres acentos característicos del español (agudo -A-, llano -LL-, y esdrújulo -E-). El resultado de la notación prosódica de los dos primeros acentos tonales de la oración es el que se muestra en las tablas 7-9.

\begin{tabular}{|c|c|c|c|c|c|c|c|}
\hline \multirow{2}{*}{\multicolumn{2}{|c|}{ INFORMANTES }} & \multicolumn{7}{|c|}{ NÚCLEO AGUDO } \\
\cline { 2 - 8 } & $\mathbf{N}$ & Exp A & N & Exp LL & N & Exp E \\
\hline \multirow{2}{*}{$\begin{array}{c}\text { Zona urbana } \\
\text { sin estudios }\end{array}$} & WCo1 & $\mathrm{L}+>\mathrm{H}^{*}$ & $\mathrm{~L}^{*}+\mathrm{H}$ & $\mathrm{L}+\mathrm{H}^{*}$ & $¡ \mathrm{H}^{*}$ & $\mathrm{~L}+>\mathrm{H}^{*}$ & $\mathrm{H}^{*}$ \\
\cline { 2 - 8 } & $\mathrm{WCo} 2$ & $\mathrm{~L}+\mathrm{H}$ & $\mathrm{H}^{*}$ & $\mathrm{~L}+>\mathrm{H}^{*}$ & $\mathrm{H}^{*}$ & $\mathrm{~L}+>\mathrm{H}^{*}$ & $\mathrm{H}^{*}$ \\
\hline \multirow{2}{*}{$\begin{array}{c}\text { Zona rural } \\
\text { sin estudios }\end{array}$} & $\mathrm{WCo} 3$ & $\mathrm{~L}+>\mathrm{H}^{*}$ & $\mathrm{H}^{*}$ & $\mathrm{~L}+>\mathrm{H}^{*}$ & $\mathrm{H}^{*}$ & $\mathrm{~L}+>\mathrm{H}^{*}$ & $\mathrm{H}^{*}$ \\
\cline { 2 - 8 } & $\mathrm{WCo} 4$ & $\mathrm{~L}+\mathrm{H}^{*}$ & $\mathrm{H}^{*}$ & $\mathrm{~L}+\mathrm{H}^{*}$ & $\mathrm{H}^{*}$ & $\mathrm{~L}+>\mathrm{H}^{*}$ & $\mathrm{H}^{*}$ \\
\hline $\begin{array}{c}\text { Zona urbana } \\
\text { con estudios }\end{array}$ & $\mathrm{WCo5}$ & $\mathrm{L}+>\mathrm{H}^{*}$ & $\mathrm{H}^{*}$ & $\mathrm{~L}+>\mathrm{H}^{*}$ & $\mathrm{H}+\mathrm{L}^{*}$ & $\mathrm{~L}+\mathrm{H}^{*}$ & $\mathrm{H}^{*}$ \\
\cline { 2 - 8 } & $\mathrm{WCo} 6$ & $\mathrm{~L}+>\mathrm{H}^{*}$ & $\mathrm{H}+\mathrm{L}^{*}$ & $\mathrm{~L}+\mathrm{H}^{*}$ & $\mathrm{H}^{*}$ & $\mathrm{~L}+>\mathrm{H}^{*}$ & $\mathrm{H}^{*}$ \\
\hline
\end{tabular}

Tabla 7

\begin{tabular}{|c|c|c|c|c|c|c|c|}
\hline \multirow{2}{*}{\multicolumn{2}{|c|}{ INFORMANTES }} & \multicolumn{6}{|c|}{ NÚCLEO LLANO } \\
\hline & & \multirow{2}{*}{$\frac{\mathbf{N}}{\mathrm{L}^{*+\mathrm{H}}}$} & \multirow{2}{*}{$\frac{\operatorname{Exp} A}{\mathrm{~L}^{*}+\mathrm{H}}$} & \multirow{2}{*}{$\begin{array}{c}\mathbf{N} \\
\mathrm{L}^{*}+\mathrm{H}\end{array}$} & \multirow{2}{*}{$\begin{array}{c}\operatorname{Exp} \mathbf{L L} \\
\mathrm{L}^{*}+\mathrm{H}\end{array}$} & \multirow{2}{*}{$\frac{\mathbf{N}}{\mathrm{L}^{*}+\mathrm{H}}$} & \multirow{2}{*}{$\frac{\operatorname{Exp} \mathbf{E}}{H^{*}}$} \\
\hline Zona urbana & WCo1 & & & & & & \\
\hline sin estudios & WCo2 & $\mathrm{L}+>\mathrm{H}^{*}$ & $\mathrm{~L}^{*+\mathrm{H}}$ & $\mathrm{L}+>\mathrm{H}^{*}$ & $\mathrm{H}^{*}$ & $\mathrm{~L}+>\mathrm{H}^{*}$ & $\mathrm{H}^{*}$ \\
\hline \multirow{2}{*}{$\begin{array}{l}\text { Zona rural } \\
\text { sin estudios }\end{array}$} & WCo3 & $\mathrm{L}^{*+\mathrm{H}}$ & $\mathrm{H}^{*}$ & $\mathrm{~L}+>\mathrm{H}^{*}$ & $\mathrm{H}^{*}$ & $\mathrm{~L}+>\mathrm{H}^{*}$ & $\mathrm{H}^{*}$ \\
\hline & WCo4 & $\mathrm{L}+>\mathrm{H}^{*}$ & $\mathrm{~L}^{*+\mathrm{H}}$ & $\mathrm{L}+\mathrm{H}^{*}$ & $\mathrm{H}^{*}$ & $\mathrm{~L}+>\mathrm{H}^{*}$ & $\mathrm{H}^{*}$ \\
\hline \multirow{2}{*}{$\begin{array}{l}\text { Zona urbana } \\
\text { con estudios }\end{array}$} & WCo5 & $\mathrm{L}+>\mathrm{H}^{*}$ & $\mathrm{H}^{*}$ & $\mathrm{~L}+>\mathrm{H}^{*}$ & $\mathrm{H}^{*}$ & $\mathrm{~L}+>\mathrm{H}^{*}$ & $\mathrm{H}^{*}$ \\
\hline & WCo6 & $\mathrm{L}+>\mathrm{H}^{*}$ & $\mathrm{~L}+\mathrm{H}^{*}$ & $\mathrm{~L}+>\mathrm{H}^{*}$ & $\mathrm{~L}^{*}+\mathrm{H}$ & $\mathrm{L}+>\mathrm{H}^{*}$ & $\mathrm{~L}^{*}+\mathrm{H}$ \\
\hline
\end{tabular}

Tabla 8 


\begin{tabular}{|c|c|c|c|c|c|c|c|}
\hline \multirow{2}{*}{\multicolumn{2}{|c|}{ INFORMANTES }} & \multicolumn{6}{|c|}{ NÚCLEO ESDRÚJULO } \\
\hline & & \multirow{2}{*}{$\frac{\mathbf{N}}{\mathrm{L}+>\mathrm{H}^{*}}$} & \multirow{2}{*}{$\frac{\operatorname{Exp} \mathbf{A}}{L+>_{i} H^{*}}$} & \multirow{2}{*}{$\frac{\mathbf{N}}{\mathrm{L}+>\mathrm{H}^{*}}$} & \multirow{2}{*}{$\begin{array}{c}\operatorname{Exp} \mathbf{L L} \\
\mathrm{L}^{*}+\mathrm{H} \\
\end{array}$} & \multirow{2}{*}{$\frac{\mathbf{N}}{\mathrm{L}+>\mathrm{H}^{*}}$} & \multirow{2}{*}{$\frac{\operatorname{Exp} \mathbf{E}}{\mathrm{H}^{*}}$} \\
\hline Zona urbana & WCo1 & & & & & & \\
\hline sin estudios & WCo2 & $\mathrm{L}+>\mathrm{H}^{*}$ & $\mathrm{~L}+\mathrm{H}^{*}$ & $\mathrm{~L}+>\mathrm{H}^{*}$ & $\mathrm{H}^{*}$ & $\mathrm{~L}+>\mathrm{H}^{*}$ & $\mathrm{H}^{*}$ \\
\hline \multirow{2}{*}{$\begin{array}{c}\text { Zona rural } \\
\text { sin estudios }\end{array}$} & WCo3 & $\mathrm{L}+>\mathrm{H}^{*}$ & $\mathrm{~L}^{*}+\mathrm{H}$ & $\mathrm{L}+>\mathrm{H}^{*}$ & $\mathrm{H}^{*}$ & $\mathrm{~L}+>\mathrm{H}^{*}$ & $\mathrm{H}^{*}$ \\
\hline & WCo4 & $\mathrm{L}+>\mathrm{H}^{*}$ & $\mathrm{~L}^{*}+\mathrm{H}$ & $\mathrm{L}+>\mathrm{H}^{*}$ & $\mathrm{~L}+>\mathrm{H}^{*}$ & $\mathrm{~L}+>\mathrm{H}^{*}$ & $\mathrm{H}^{*}$ \\
\hline \multirow{2}{*}{$\begin{array}{l}\text { Zona urbana } \\
\text { con estudios }\end{array}$} & WCo5 & $\mathrm{L}+>\mathrm{H}^{*}$ & $\mathrm{H}^{*}$ & $\mathrm{~L}+>\mathrm{H}^{*}$ & $\mathrm{H}^{*}$ & $\mathrm{~L}+>\mathrm{H}^{*}$ & $\mathrm{H}^{*}$ \\
\hline & WCo6 & $\mathrm{L}+>\mathrm{H}^{*}$ & $\mathrm{~L}+\mathrm{H}^{*}$ & $\mathrm{~L}+>\mathrm{H}^{*}$ & $\mathrm{~L}^{*}+\mathrm{H}$ & $\mathrm{L}+>\mathrm{H}^{*}$ & $\mathrm{~L}^{*}+\mathrm{H}$ \\
\hline
\end{tabular}

Tabla 9

Como se observa en las tablas, el primer acento de las declarativas es ascendente con el pico desplazado a la postónica $\left[\mathrm{L}+>\mathrm{H}^{*}\right]$ en prácticamente todos los casos $(79,6 \%)$. Esta realización, como hemos visto para las declarativas sin expansión, la consideramos variante del contorno bitonal $/ \mathrm{L}+\mathrm{H}^{*} /$ puesto que lo relevante es que el ascenso inicial de la $\mathrm{F}_{0}$ alcance la tónica aunque culmine después de esta; solo puntualmente el ascenso inicial se origina después de la tónica $([\mathrm{L} *+\mathrm{H}] 9,3 \%)$.

La expansión, en cambio, presenta frecuentemente un acento tonal alto $\left(/ \mathrm{H}^{*} /\right)$ que se manifiesta con variantes o estructuras superficiales monotonales $\left(\left[\mathrm{H}^{*}\right]\right.$ o $\left.\left[{ }_{i} \mathrm{H}^{*}\right]\right)$ o bitonales $\left(\left[\mathrm{L}+\mathrm{H}^{*}\right] ;\left[\mathrm{L}+>\mathrm{H}^{*}\right] ;\left[\mathrm{L}+>_{i} \mathrm{H}^{*}\right]\right)$ puesto que la $\mathrm{F}_{0}$ asciende de manera brusca en el primer elemento del sintagma (núcleo sintagmático) estabilizando su movimiento en la expansión; por ello, en pocos casos el segundo ascenso de la frecuencia presenta un valle con suficiente profundidad ( $\geq 1,5 \mathrm{st}$ ) como para que podamos etiquetar $/ \mathrm{L} /$ en la estructura profunda $\left(\left[\mathrm{L}+\mathrm{H}^{*}\right]\right.$ $5,6 \% ;\left[\mathrm{L}+>\mathrm{H}^{*}\right] 1,8 \%$ o [L+> $\left.\left.\mathrm{H}^{*}\right] 1,8 \%\right)$ o que la tónica se encuentre baja $(/ \mathrm{L} * /:[\mathrm{L} *+\mathrm{H}] 22,2$ $\%$; $\left[\mathrm{H}+\mathrm{L}^{*}\right] 3,7 \%$ ). En la tabla 10 podemos ver un resumen de lo expuesto en las tablas 7-9.

\begin{tabular}{|c|c|c|c|}
\hline \multicolumn{4}{|c|}{ Invariantes y variantes } \\
\hline \multicolumn{4}{|c|}{ SN } \\
\hline \multirow{2}{*}{\multicolumn{2}{|c|}{$\begin{array}{c}\mathrm{N} \\
\text { Acento } 1 \\
\end{array}$}} & \multicolumn{2}{|c|}{$\operatorname{Exp}$} \\
\hline & & \multicolumn{2}{|c|}{ Acento 2} \\
\hline I & $\mathbf{V}$ & I & $\mathbf{V}$ \\
\hline $\mathrm{L}+\mathrm{H}^{*}$ & $\begin{array}{c}\mathrm{L}+\mathrm{H}^{*} 11,1 \% \\
\mathrm{~L}+>\mathrm{H}^{*} 79,6 \%{ }^{13}\end{array}$ & $\mathrm{H}^{*}$ & $\begin{array}{c}\mathrm{H}^{*} 63 \% \\
¡ \mathrm{H}^{*} 1,8 \% \\
\mathrm{~L}+\mathrm{H}^{*} 5,6 \% \\
\mathrm{~L}+>\mathrm{H}^{*} 1,8 \% \\
\mathrm{~L}+>_{i} \mathrm{H}^{*} 1,8 \%{ }^{14}\end{array}$ \\
\hline
\end{tabular}

Tabla 10

13 El 9,3\% restante que falta en la casilla corresponde al etiquetaje [L*+H].

14 El 26\% restante corresponde al etiquetaje $\left[\mathrm{L}^{*}+\mathrm{H}\right](22,2 \%)$ y $\left[\mathrm{H}+\mathrm{L}^{*}\right](3,7 \%)$ realizaciones más esporádicas. 
En definitiva, el núcleo del sintagma nominal se caracteriza por presentar en su estructura profunda un acento ascendente $\left(/ \mathrm{L}+\mathrm{H}^{*} /\right)$ seguido de un acento alto en la sílaba tónica $\left(/ \mathrm{H}^{*} /\right)$ durante la expansión, tal como reflejamos en la tabla 11 e ilustramos en las figuras 12 y 13 .

\begin{tabular}{|c|c|}
\hline \multicolumn{2}{|c|}{ Invariantes } \\
\hline \multicolumn{2}{|c|}{ SN } \\
\hline $\mathbf{N}$ & $\mathbf{E x p}$ \\
\hline $\mathrm{L}+\mathrm{H}^{*}$ & $\mathrm{H}^{*}$ \\
\hline
\end{tabular}

Tabla 11

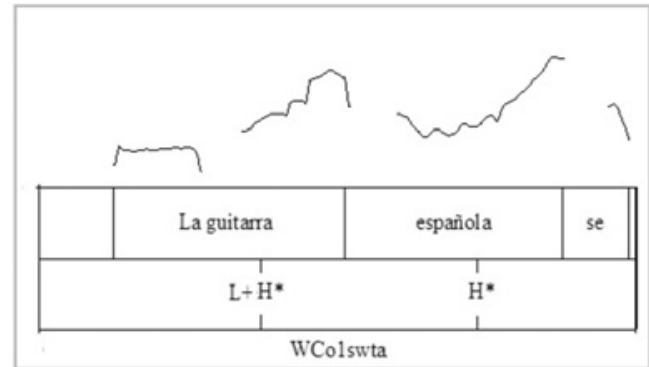

WCo1 (Sexo femenino)
Figuras 12-13

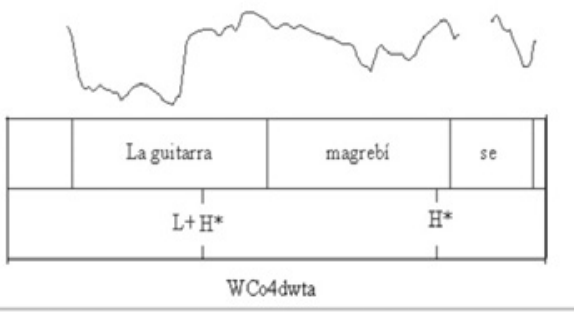

WCo4 (Sexo masculino)

b) Las tablas 12-14 reflejan la interpretación de los dos últimos acentos tonales de las declarativas con expansión en el objeto y el tono de frontera final de los seis informantes. La tabla 15 resume las invariantes que aparecen en dichas tablas, así como sus realizaciones con el correspondiente porcentaje de aparición.

\begin{tabular}{|c|c|c|c|c|c|c|c|}
\hline \multirow{2}{*}{\multicolumn{2}{|c|}{ INFORMANTES }} & \multicolumn{7}{|c|}{ NÚCLEO AGUDO } \\
\cline { 2 - 8 } & N & Exp A & N & Exp LL & N & Exp E \\
\hline \multirow{2}{*}{$\begin{array}{c}\text { Zona urbana } \\
\text { sin estudios }\end{array}$} & WCo1 & $\mathrm{L}+>\mathrm{H}^{*}$ & $\mathrm{~L} * \mathrm{~L} \%$ & $\mathrm{~L}+! \mathrm{H}^{*}$ & $\mathrm{~L} * \mathrm{~L} \%$ & $\mathrm{~L}+! \mathrm{H}^{*}$ & $\mathrm{~L} * \mathrm{~L} \%$ \\
\cline { 2 - 8 } & $\mathrm{WCo} 2$ & $\mathrm{~L}+\mathrm{H}^{*}$ & $\mathrm{~L} * \mathrm{~L} \%$ & $\mathrm{~L}+>\mathrm{H}^{*}$ & $\mathrm{~L} * \mathrm{~L} \%$ & $\mathrm{~L}+\mathrm{H}^{*}$ & $\mathrm{~L} * \mathrm{~L} \%$ \\
\hline $\begin{array}{c}\text { Zona rural } \\
\text { sin estudios }\end{array}$ & $\mathrm{WCo} 3$ & $\mathrm{~L}+>\mathrm{H}^{*}$ & $\mathrm{~L} * \mathrm{~L} \%$ & $\mathrm{~L}+>\mathrm{H}^{*}$ & $\mathrm{~L} * \mathrm{~L} \%$ & $\mathrm{~L} *$ & $\mathrm{~L} * \mathrm{~L} \%$ \\
\cline { 2 - 8 } & $\mathrm{WCo} 4$ & $\mathrm{~L} * \mathrm{H}$ & $\mathrm{L} * \mathrm{~L} \%$ & $\mathrm{~L}+\mathrm{H}^{*}$ & $\mathrm{~L} * \mathrm{~L} \%$ & $\mathrm{~L}+! \mathrm{H}^{*}$ & $\mathrm{~L} * \mathrm{~L} \%$ \\
\hline $\begin{array}{c}\text { Zona urbana } \\
\text { con estudios }\end{array}$ & $\mathrm{WCo} 5$ & $\mathrm{~L}+\mathrm{H}^{*}$ & $\mathrm{~L} * \mathrm{~L} \%$ & $\mathrm{~L}+\mathrm{H}^{*}$ & $\mathrm{~L} * \mathrm{~L} \%$ & $\mathrm{~L}+\mathrm{H}^{*}$ & $\mathrm{~L} * \mathrm{~L} \%$ \\
\cline { 2 - 8 } & $\mathrm{WCo} 6$ & $\mathrm{~L}+! \mathrm{H}^{*}$ & $\mathrm{~L} * \mathrm{~L} \%$ & $\mathrm{~L}+! \mathrm{H}^{*}$ & $\mathrm{~L} * \mathrm{~L} \%$ & $\mathrm{~L}+\mathrm{H}^{*}$ & $\mathrm{~L} * \mathrm{~L} \%$ \\
\hline
\end{tabular}

Tabla 12 


\begin{tabular}{|c|c|c|c|c|c|c|c|}
\hline \multirow{2}{*}{ INFORMANTES } & \multicolumn{7}{|c|}{ NÚCLEO LLANO } \\
\cline { 2 - 8 } & N & Exp A & N & Exp LL & N & Exp E \\
\hline \multirow{2}{*}{$\begin{array}{c}\text { Zona urbana } \\
\text { sin estudios }\end{array}$} & $\mathrm{WCo} 1$ & $\mathrm{~L}+>\mathrm{H}^{*}$ & $\mathrm{~L} * \mathrm{~L} \%$ & $\mathrm{~L} *+\mathrm{H}$ & $\mathrm{L} * \mathrm{~L} \%$ & $\mathrm{~L} *+\mathrm{H}$ & $\mathrm{L} * \mathrm{~L} \%$ \\
\cline { 2 - 8 } & $\mathrm{WCo} 2$ & $\mathrm{H}^{*}$ & $\mathrm{~L} * \mathrm{~L} \%$ & $\mathrm{H}^{*}$ & $\mathrm{~L} * \mathrm{~L} \%$ & $\mathrm{H}^{*}$ & $\mathrm{~L} * \mathrm{~L} \%$ \\
\hline $\begin{array}{c}\text { Zona rural } \\
\text { sin estudios }\end{array}$ & $\mathrm{WCo} 3$ & $\mathrm{~L}+>\mathrm{H}^{*}$ & $\mathrm{~L} * \mathrm{~L} \%$ & $\mathrm{~L} *+\mathrm{H}$ & $\mathrm{L} * \mathrm{~L} \%$ & $\mathrm{~L}+>\mathrm{H}^{*}$ & $\mathrm{~L} * \mathrm{~L} \%$ \\
\cline { 2 - 8 } & $\mathrm{WCo} 4$ & $\mathrm{~L}+>\mathrm{H}^{*}$ & $\mathrm{~L} * \mathrm{~L} \%$ & $\mathrm{~L} *+\mathrm{H}$ & $\mathrm{L} * \mathrm{~L} \%$ & $\mathrm{~L} *$ & $\mathrm{~L} * \mathrm{~L} \%$ \\
\hline $\begin{array}{c}\text { Zona urbana } \\
\text { con estudios }\end{array}$ & $\mathrm{WCo} 5$ & $\mathrm{~L}+\mathrm{H}^{*}$ & $\mathrm{~L} * \mathrm{~L} \%$ & $\mathrm{~L}+>\mathrm{H}^{*}$ & $\mathrm{~L} * \mathrm{~L} \%$ & $\mathrm{~L}+>\mathrm{H}^{*}$ & $\mathrm{~L} * \mathrm{~L} \%$ \\
\cline { 2 - 8 } & $\mathrm{WCo} 6$ & $\mathrm{~L}+>\mathrm{H}^{*}$ & $\mathrm{~L} * \mathrm{~L} \%$ & $\mathrm{~L}+>\mathrm{H}^{*}$ & $\mathrm{~L} * \mathrm{~L} \%$ & $\mathrm{~L}+>\mathrm{H}^{*}$ & $\mathrm{~L} * \mathrm{~L} \%$ \\
\hline
\end{tabular}

Tabla 13

\begin{tabular}{|c|c|c|c|c|c|c|c|}
\hline \multirow{2}{*}{\multicolumn{2}{|c|}{ INFORMANTES }} & \multicolumn{6}{|c|}{ NÚCLEO ESDRÚJULO } \\
\hline & & \multirow{2}{*}{$\frac{\mathbf{N}}{\mathrm{L}^{*+}{ }_{i} \mathrm{H}}$} & \multirow{2}{*}{$\begin{array}{l}\operatorname{Exp} A \\
L * L \% \\
\end{array}$} & \multirow{2}{*}{$\frac{\mathbf{N}}{\mathrm{L}^{*}+\mathrm{H}}$} & \multirow{2}{*}{$\frac{\operatorname{Exp} \mathbf{L L}}{\mathrm{L} * \mathrm{~L} \%}$} & \multirow{2}{*}{$\frac{\mathbf{N}}{\mathrm{L}^{*}+\mathrm{H}}$} & \multirow{2}{*}{$\begin{array}{l}\operatorname{Exp} \mathbf{E} \\
* L \%\end{array}$} \\
\hline Zona urbana & WCo1 & & & & & & \\
\hline sin estudios & WCo2 & $\mathrm{L}^{*}+\mathrm{H}$ & $\mathrm{L} * \mathrm{~L} \%$ & $\mathrm{H}^{*}$ & $\mathrm{~L} * \mathrm{~L} \%$ & $\mathrm{H}^{*}$ & $\mathrm{~L} * \mathrm{~L} \%$ \\
\hline \multirow{2}{*}{$\begin{array}{l}\text { Zona rural sin } \\
\text { estudios }\end{array}$} & WCo3 & $\mathrm{H}^{*}$ & $\mathrm{~L} * \mathrm{~L} \%$ & $\mathrm{H}^{*}$ & $\mathrm{~L} * \mathrm{~L} \%$ & $\mathrm{H}^{*}$ & $\mathrm{~L} * \mathrm{~L} \%$ \\
\hline & WCo4 & $\mathrm{H}^{*}$ & $\mathrm{~L} * \mathrm{~L} \%$ & $\mathrm{~L} *+\mathrm{H}$ & $\mathrm{L} * \mathrm{~L} \%$ & $\mathrm{H}^{*}$ & $\mathrm{~L} * \mathrm{~L} \%$ \\
\hline \multirow{2}{*}{$\begin{array}{l}\text { Zona urbana } \\
\text { con estudios }\end{array}$} & WCo5 & $\mathrm{L}+>_{i} \mathrm{H}^{*}$ & $\mathrm{~L} * \mathrm{~L} \%$ & $\mathrm{~L}+>\mathrm{H}^{*}$ & $\mathrm{~L} * \mathrm{~L} \%$ & $\mathrm{~L}+>\mathrm{H}^{*}$ & $\mathrm{~L} * \mathrm{~L} \%$ \\
\hline & WCo6 & $\mathrm{H}^{*}$ & $\mathrm{~L} * \mathrm{~L} \%$ & $\mathrm{~L} *+\mathrm{H}$ & $\mathrm{L} * \mathrm{~L} \%$ & $\mathrm{~L}^{*}+\mathrm{H}$ & $\mathrm{L} * \mathrm{~L} \%$ \\
\hline
\end{tabular}

Tabla 14

\begin{tabular}{|c|c|c|c|c|c|}
\hline \multicolumn{6}{|c|}{ Invariantes y variantes } \\
\hline \multicolumn{6}{|c|}{ SPrep } \\
\hline \multirow{2}{*}{\multicolumn{2}{|c|}{$\frac{\mathrm{N}}{\text { Acento } 3}$}} & \multicolumn{4}{|c|}{ Exp } \\
\hline & & \multicolumn{2}{|c|}{ Acento 4} & \multicolumn{2}{|c|}{$\%$} \\
\hline $\mathbf{I}$ & $\mathbf{V}$ & I & $\mathbf{V}$ & I & $\mathbf{V}$ \\
\hline $\mathbf{H}^{*}$ & $\begin{array}{c}\mathrm{H}^{*} 20,4 \% \\
\mathrm{~L}+\mathrm{H}^{*} 14,8 \% \\
\mathrm{~L}+! \mathrm{H}^{*} 9,3 \% \\
\mathrm{~L}+\mathrm{H}^{*} 25,9 \% \\
\mathrm{~L}+>_{i} \mathrm{H}^{*} 3,7 \% 15\end{array}$ & $\mathbf{L}^{*}$ & $\mathrm{~L}^{*} 100 \%$ & $\mathrm{~L} \%$ & $\mathrm{~L} \% 100 \%$ \\
\hline
\end{tabular}

Tabla 15

15 El $25,9 \%$ restante corresponde al etiquetaje $\left[\mathrm{L}^{*}\right](3,7 \%),\left[\mathrm{L}^{*+} \mathrm{H}\right](20,4 \%)$ y $\left[\mathrm{L}^{*+}{ }_{\mathrm{i}} \mathrm{H}\right](1,8 \%)$ realizaciones más esporádicas. 
En las tablas precedentes puede verse que la expansión del objeto constituye ahora el acento nuclear de la frase que sigue asociándose a un tono bajo $\left(/ \mathrm{L}^{*} /\right)$ seguido de un tono de frontera igualmente bajo (/L\%/). Por tanto, sigue manteniéndose la configuración tonemática de las oraciones más breves: $/ \mathrm{L} * \mathrm{~L} \% /$. La diferencia la plantea el último acento prenuclear (tercero de la oración) que en este tipo de oraciones es el núcleo del sintagma preposicional. Ya veíamos en el apartado de las declarativas sin expansión (5.1.1., tabla 4) que si bien la invariante detectada en el SV se caracterizaba por presentar generalmente un acento bajo en la sílaba tónica -to- (se toca $\left./ \mathrm{L}^{*} /\left[\mathrm{L}^{*}\right]\right)$, también podía presentarse un tono bajo seguido de un

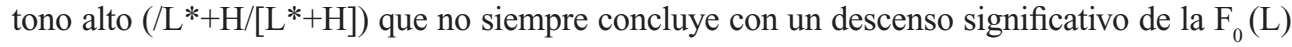
generando la estructura superficial $\left[(\mathrm{L}+) \mathrm{H}^{*}\right]$. De ahí que la forma invariante que proponemos para el tercer acento sea $/ \mathrm{H}^{*} /$ y no $/ \mathrm{L}+\mathrm{H}^{*} /$, es decir, lo frecuente en este punto de la curva (último acento prenuclear) es que la tónica quede alta como consecuencia del mantenimiento del tono desde el acento anterior o por un nuevo ascenso de la frecuencia fundamental; solo ocasionalmente (en un 25,9\%) encontramos una tónica baja $\left(\left[\mathrm{L}^{*}\right] ;[\mathrm{L} *+\mathrm{H}] ;\left[\mathrm{L}^{*}+{ }_{j} \mathrm{H}\right]\right.$ ).

Teniendo en cuenta lo expuesto, nuestra propuesta para los dos últimos acentos de las declarativas con expansión en el objeto es la que reflejamos en la tabla 16 e ilustramos en las figuras 14 y 15 donde se puede ver la trayectoria de la F0 en dichos acentos.

\begin{tabular}{|c|c|}
\hline \multicolumn{2}{|c|}{ Invariantes } \\
\hline \multicolumn{2}{|c|}{ SPrep } \\
\hline $\mathbf{N}$ & $\mathbf{E x p}$ \\
\hline $\mathrm{H}^{*}$ & $\mathrm{~L}^{*} \mathrm{~L} \%$ \\
\hline
\end{tabular}

Tabla 16

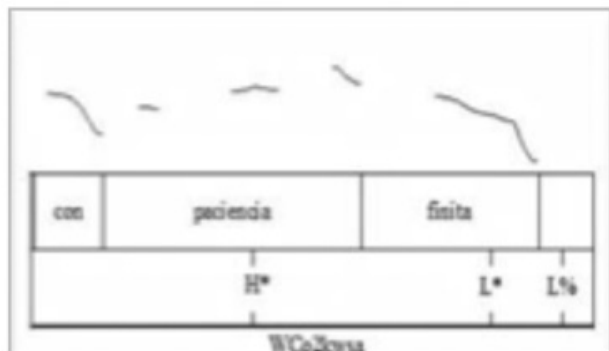

WCo2 (Sexo femenino)

Figuras 14-15

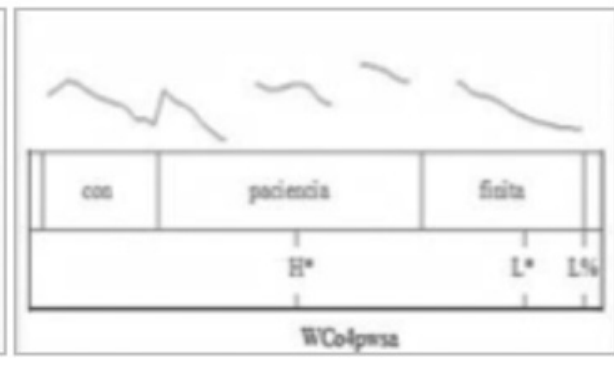

WCo4 (Sexo masculino)

\subsection{Corpus semiespontáneo y espontáneo}

\subsubsection{Oraciones con un solo pico máximo}

Después de analizar los intervalos de F0 en el corpus situacional o semiespontáneo (CS) y comprobar si superan o no el umbral perceptivo de $1,5 \mathrm{st}$, ofrecemos en la tabla 17 los resultados globales del etiquetaje de los acentos tonales inicial y nuclear, así como el tono 
de frontera final de las curvas. Reflejamos en ella solo las invariantes más frecuentes con sus respectivas variantes y los porcentajes de aparición de estas últimas.

\begin{tabular}{|c|c|c|c|c|c|}
\hline \multicolumn{5}{|c|}{ Invariantes y variantes } \\
\hline \multicolumn{2}{|c|}{ ACENTO INICIAL ${ }^{\mathbf{1 6}}$} & \multicolumn{2}{|c|}{ ACENTO NUCLEAR } & \multicolumn{1}{c|}{} \\
\hline I & $\mathbf{V}$ & $\mathbf{I}$ & $\mathbf{V}$ & I & V \\
\hline $\mathbf{L}^{*}+\mathbf{H}$ & $\mathrm{L}^{*}+\mathrm{H} 50 \%{ }^{17}$ & $\mathbf{L}^{*}$ & $\begin{array}{c}\mathrm{L}^{*} 94,7 \% \\
\mathrm{H}+\mathrm{L}^{*} 2,6 \%\end{array}$ & $\mathbf{L} \%$ & $\begin{array}{c}\mathrm{L} \% 70,3 \% \\
\mathrm{ML} \% 24,3 \%\end{array}$ \\
\hline
\end{tabular}

Tabla 17

Según nuestros resultados, en las declarativas monocumbres existe variabilidad en el primer acento, aunque el porcentaje mayor es el de $/ \mathrm{L} *+\mathrm{H} /(50 \%)$ reflejado en la tabla debido, en parte, a la variación silábica de las palabras en posición inicial ${ }^{20}$. Menores porcentajes se registran para el contorno ascendente bitonal $\left(/ \mathrm{L}+\mathrm{H}^{*} /\right)$ que puede realizarse con el pico desplazado a la postónica ([L+> $\left.\left.\mathrm{H}^{*}\right] 33,3 \%\right)$ o dentro de los límites de la tónica $\left(\left[\mathrm{L}+\mathrm{H}^{*}\right]\right.$ $5,6 \%)$. Puntualmente puede llegar a aparecer la variante monotonal $\left[\mathrm{H}^{*}\right](11,1 \%)$ debido a que el rango del pico inicial no alcanza el semitono y medio.

El último acento es frecuentemente bajo en la tónica $\left(/ \mathrm{L}^{*} /\right)$ seguido de un tono bajo (/L\%/ [L\%]; [ML\%] 94,6\%); puntualmente encontramos un tono de frontera alto o medio ([H\%] 2,7\%; [M\%] 2,7\%). No obstante, cuando la frase solo tiene un acento se presentan dos alternativas antes del descenso final: la primera es que se dé un acento descendente durante la tónica precedida de un tono alto $\left(\left[\mathrm{H}+\mathrm{L}^{*}\right] 2,6 \%\right)$ por lo que seguiríamos estando ante una variante de $/ \mathrm{L} *$; la segunda opción es que, debido al escaso espacio por la reducción de los acentos, la tónica presente un tono alto $\left(\left[\mathrm{H}^{*}\right] 2,6 \%\right)$. Estas dos alternativas están ligadas a la producción y no son características dialectales puesto que si ampliáramos el número de acentos se generaría un contorno tonal descendente durante la tónica.

Por otra parte, en el corpus espontáneo o map task $(C E)$ se da un movimiento ascendente en el acento inicial que incide mayoritariamente en la acentuada $\left(/ \mathrm{L}+\mathrm{H}^{*} /\right)$; en algunos casos la $\mathrm{F}_{0}$ continúa ascendiendo $\left(\left[\mathrm{L}+>\mathrm{H}^{*}\right] 11,1 \%\right)$. En menores ocasiones el ascenso es bajo en la tónica seguido de un tono alto $\left(\left[\mathrm{L}^{*}+\mathrm{H}\right] 33,3 \%\right)$ y, más esporádicamente aún, un tono sostenido ([H*] 22,2\%). Véase la tabla 18.

16 Excluimos del acento inicial las frases con un solo acento puesto que la configuración tonal de este tipo de oraciones corresponde, como hemos visto en la descripción fonética, con el núcleo entonativo.

17 El $50 \%$ restante corresponde al etiquetaje $/ \mathrm{H}^{*} /\left(\left[\mathrm{H}^{*}\right] 11,1 \%\right) ; / \mathrm{L}+\mathrm{H}^{*} /\left(\left[\mathrm{L}+\mathrm{H}^{*}\right]\right.$ 5,6 \% y $\left[\mathrm{L}+>\mathrm{H}^{*}\right] 33,3$ $\%$; invariantes con menor porcentaje de aparición de sus variantes.

18 El 2,6 \% restante corresponde al etiquetaje [ $\left.\mathrm{H}^{*}\right]$ (de la invariante $/ \mathrm{H}^{*} /$ ) realización esporádica como indica el propio porcentaje en las oraciones con un solo acento. Recordemos que, como indicamos en la descripción fonética, la solución de este tipo de declarativas es el alineamiento tonal del pico en el único acento de la frase $\left(\left[\mathrm{H}^{*}\right]\right)$ o el adelantamiento a la pretónica $\left(\left[\mathrm{H}+\mathrm{L}^{*}\right] / \mathrm{L}^{*} /\right)$.

19 El porcentaje restante corresponde a [M\%] 2,7 \% y [H\%] 2,7 \% variantes de /M\%/ y /H\%/, respectivamente.

20 Es evidente que el corpus más espontáneo, frente al experimental que contaba con trisílabos iniciales, no tiene un número de sílabas fijo en posición inicial y es frecuente encontrar palabras llanas con dos sílabas como por ejemplo Hace, cobro, carne, etc. Ello facilita que la posición de la tónica se encuentre baja en algunas oraciones. Pero como veremos en la tabla 20, si consideramos conjuntamente los dos tipos de corpus (semiespontáneo y espontáneo) en las oraciones monocumbres, el esquema $/ \mathrm{L} * \mathrm{H} /$ alterna con $/ \mathrm{L}+\mathrm{H}^{*} /$. 


\begin{tabular}{|c|c|c|c|c|c|}
\hline \multicolumn{6}{|c|}{ Invariantes y variantes } \\
\hline \multicolumn{2}{|c|}{ ACENTO INICIAL ${ }^{\mathbf{2 1}}$} & \multicolumn{2}{|c|}{ ACENTO NUCLEAR } & \multicolumn{2}{c|}{} \\
\hline I & $\mathbf{V}$ & I & V & I & V \\
\hline L+H* & $\begin{array}{c}\mathrm{L}+\mathrm{H}^{*} 33,3 \% \\
\mathrm{~L}+>\mathrm{H}^{*} 11,1 \% \%^{22}\end{array}$ & $\mathbf{L}^{*}$ & $\mathrm{~L}^{*} 92,9 \% \%^{23}$ & $\mathrm{~L} \%$ & $\begin{array}{c}\mathrm{L} \% 73,3 \% \\
\mathrm{ML} \% 13,3 \%{ }^{24}\end{array}$ \\
\hline
\end{tabular}

Tabla 18

En el núcleo final, se registra un descenso de la frecuencia $(/ \mathrm{L} * /)$ seguido de un tono bajo (/L\%/ [L\%]; [ML\%] 86,6 \%) si la oración tiene más de un acento; si tiene solo uno, como en el corpus semiespontáneo, se puede dar un tono alto durante la tónica $\left(\left[\mathrm{H}^{*}\right] 7,1 \%\right)$ antes del descenso final.

A partir de los resultados obtenidos en las oraciones declarativas con un solo pico tonal en los dos tipos de corpus ( $C S$ y $C E$ ), hemos realizado los promedios de los porcentajes de realización en las distintas variantes o estructuras superficiales con el objetivo de obtener el mayor número de realizaciones posibles a la hora de determinar las invariantes o estructuras profundas representativas de los acentos de frontera. Véanse los resultados en la tabla 19.

\begin{tabular}{|c|c|c|c|c|c|}
\hline \multicolumn{6}{|c|}{ Invariantes y variantes } \\
\hline \multicolumn{2}{|c|}{ ACENTO INICIAL ${ }^{25}$} & \multicolumn{2}{|c|}{ ACENTO NUCLEAR } & \multicolumn{2}{|c|}{ TONO DE FRONTERA\% } \\
\hline $\mathbf{I}$ & $\mathbf{V}$ & I & $\mathbf{V}$ & $\mathbf{I}$ & $\mathbf{V}$ \\
\hline $\mathbf{L}+\mathbf{H}^{*}$ & $\begin{array}{c}\mathrm{L}+\mathrm{H}^{*} 19,4 \% \\
\mathrm{~L}+>\mathrm{H}^{*} 22,2 \%\end{array}$ & \multirow[t]{2}{*}{$\mathbf{L}^{*}$} & \multirow{2}{*}{$\begin{array}{c}\mathrm{L} * 93,7 \% \\
\mathrm{H}+\mathrm{L} * 1,3 \%{ }^{26}\end{array}$} & \multirow[t]{2}{*}{ L\% } & \multirow{2}{*}{$\begin{array}{c}\text { L\% 71,8 \% } \\
\text { ML\% 8,8 \% }\end{array}$} \\
\hline $\mathbf{L}^{*}+\mathbf{H}$ & $\mathrm{L}^{*}+\mathrm{H} 41,6 \%{ }^{28}$ & & & & \\
\hline
\end{tabular}

Tabla 19

Según estos resultados, el acento inicial de las declarativas con un solo pico máximo muestra la coexistencia de dos invariantes: $/ \mathrm{L}+\mathrm{H}^{*} / \mathrm{y} / \mathrm{L} *+\mathrm{H} /$. En cambio, el nuclear es siempre bajo $\left(/ \mathrm{L}^{*} /\right)$ seguido generalmente de un tono de frontera final, igualmente, bajo $(/ \mathrm{L} \% /$ [L\%]; [ML\%] 90,6 \%): puntualmente encontramos un tono de frontera alto o medio ([H\%] $8 \%$; [M\%] 1,4 \%). En definitiva, los acentos tonales más característicos de este tipo de oraciones son los que se incluyen en la tabla 20 ilustrados en las imágenes 16 y 17.

21 Excluimos del acento inicial las frases con un solo acento puesto que su configuración está vinculada al núcleo entonativo.

22 El 55,5\% restante corresponde al etiquetaje $\left[\mathrm{L}^{*}+\mathrm{H}\right](33,3 \%)$ y $\left[\mathrm{H}^{*}\right](22,2 \%)$ variantes de $/ \mathrm{L}^{*}+\mathrm{H} / \mathrm{y} / \mathrm{H}^{*} /$, respectivamente que tienen menor porcentaje de aparición que las variantes de $/ \mathrm{L}+\mathrm{H}^{*} /$.

23 El 7,1 \% restante corresponde al etiquetaje $\left[\mathrm{H}^{*}\right]$ (de la invariante $/ \mathrm{H}^{*} /$ ) realización esporádica como indica el propio porcentaje de las oraciones con un solo acento.

24 El porcentaje restante corresponde a [H\%] 13,3\% variante de $/ \mathrm{H} \% /$.

25 Excluimos del acento inicial las frases con un solo acento puesto que la configuración tonal de este tipo de oraciones corresponde, como hemos visto en la descripción fonética, con el núcleo entonativo.

26 El 4,8 \% restante corresponde al etiquetaje [H*] (de la invariante $/ \mathrm{H}^{*} /$ ), realización de las oraciones con un solo acento.

27 El porcentaje restante corresponde a [M\%] 1,4\% y [H\%] $8 \%$ variantes de $/ \mathrm{M} \% / \mathrm{y} / \mathrm{H} \% /$, respectivamente.

28 El 16,7 \% restante corresponde al etiquetaje $\left[\mathrm{H}^{*}\right]$ realización de $/ \mathrm{H}^{*} /$ con menor frecuencia. 


\begin{tabular}{|c|c|}
\hline \multicolumn{2}{|c|}{ Invariantes } \\
\hline ACENTO INICIAL & ACENTO NUCLEAR Y TONO DE FRONTERA \\
\hline $\mathrm{L}+\mathrm{H}^{*} / \mathrm{L} *+\mathrm{H}$ & $\mathrm{L} * \mathrm{~L} \%$ \\
\hline
\end{tabular}

Tabla 20

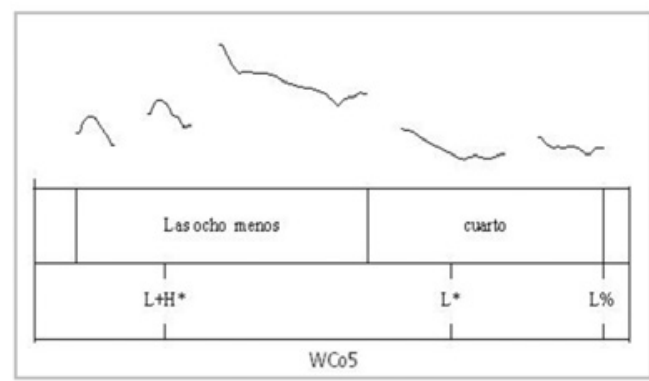

WCo5 (Sexo femenino)

Figuras 16-17

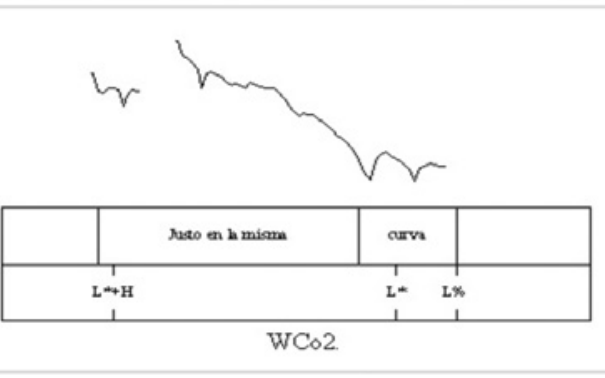

WCo2 (Sexo masculino)

\subsubsection{Oraciones con más de un pico máximo}

Mostramos en la tabla 21 los resultados obtenidos para el corpus situacional semiespontáneo $(C S)$ más extenso. La tabla contiene la interpretación de los acentos tonales inicial y nuclear y del tono de frontera final teniendo en cuenta las invariantes más frecuentes con los porcentajes de aparición de sus variantes.

\begin{tabular}{|c|c|c|c|c|c|}
\hline \multicolumn{5}{|c|}{ Invariantes y variantes } \\
\hline \multicolumn{2}{|c|}{ ACENTO INICIAL } & \multicolumn{2}{|c|}{ ACENTO NUCLEAR } & \multicolumn{2}{c|}{} \\
\hline I & $\mathbf{V}$ & I & V & I & V \\
\hline & & & & & $\mathrm{L} \% 92,9 \%$ \\
$\mathbf{L}^{*}+\mathbf{H}$ & $\mathrm{L}^{*}+\mathrm{H} 50 \% 29$ & $\mathbf{L}^{*}$ & $\mathrm{~L}^{*} 100 \%$ & $\mathbf{L} \%$ & $\mathrm{ML} \% 7,1 \%$ \\
\hline
\end{tabular}

Tabla 21

En este tipo de oraciones, el primer acento se caracteriza por presentar diferentes tendencias. Así, lo más frecuente es que el ascenso inicial se localice en la postónica por lo que la tónica se encuentra baja $\left(\left[\mathrm{L}^{*}+\mathrm{H}\right] 50 \%\right)$; no obstante, también puede darse, aunque con menor frecuencia, un acento ascendente invariante durante la tónica $\left(/ \mathrm{L}+\mathrm{H}^{*} /\right)$ que puede desplazarse o no a la postónica ([L+>H*] 7,1\%; [L+H*] 35,8\%); otra posibilidad, aunque muy puntual, es que el ascenso inicial tienda a ser un tanto sostenido $\left(\left[\mathrm{H}^{*}\right] 7,1 \%\right)$. El acento nuclear es siempre descendente $\left(/ \mathrm{L}^{*} /\right)$ y finaliza siempre con un tono de frontera bajo $(/ \mathrm{L} \% /$ [L\%]; [ML\%] $100 \%)$.

29 El $50 \%$ restante corresponde al etiquetaje $/ \mathrm{H}^{*} /\left(\left[\mathrm{H}^{*}\right]\right.$ 7,1 \%) y $/ \mathrm{L}+\mathrm{H}^{*} /\left(\left[\mathrm{L}+\mathrm{H}^{*}\right]\right.$ 35,8 \% y $\left[\mathrm{L}+>\mathrm{H}^{*}\right]$ 7,1 $\%)$; invariantes con menor porcentaje de aparición de sus variantes. 
En las oraciones procedentes del corpus espontáneo $(C E)$ se obtuvieron los resultados de la tabla 22.

\begin{tabular}{|c|c|c|c|c|c|}
\hline \multicolumn{6}{|c|}{ Invariantes y variantes } \\
\hline \multicolumn{2}{|c|}{ ACENTO INICIAL } & \multicolumn{2}{|c|}{ ACENTO NUCLEAR } & \multicolumn{2}{c|}{$\%$} \\
\hline I & $\mathbf{V}$ & $\mathbf{I}$ & $\mathbf{V}$ & $\mathbf{I}$ & V \\
\hline $\mathbf{L}+\mathbf{H}^{*}$ & $\begin{array}{c}\mathrm{L}+\mathrm{H}^{*} 25 \% \\
\mathrm{~L}+>\mathrm{H}^{*} 50 \%{ }^{30}\end{array}$ & $\mathbf{L}^{*}$ & $\mathrm{~L} * 100 \%$ & $\mathbf{L} \%$ & $\mathrm{~L} \% 100 \%$ \\
\hline
\end{tabular}

Tabla 22

En el prenúcleo, el primer acento es ascendente durante la tónica $\left(/ \mathrm{L}+\mathrm{H}^{*} /\right)$; en pocos casos el ascenso se produce después de esta $\left(\left[\mathrm{L}^{*}+\mathrm{H}\right] 25 \%\right)$. El acento nuclear, en cambio, es descendente ([L*] 100\%) y culmina con un tono de frontera bajo (/L\%/ [L\%] $100 \%)$.

En definitiva, si hallamos las medias de los porcentajes de realización de las distintas variantes en los dos tipos de corpus analizados (CS y CE) con el propósito de determinar los contornos invariantes, los resultados en las oraciones declarativas con más de un pico tonal son los que mostramos en la tabla 23.

\begin{tabular}{|c|c|c|c|c|c|}
\hline \multicolumn{6}{|c|}{ Invariantes y variantes } \\
\hline \multicolumn{2}{|c|}{ ACENTO INICIAL } & \multicolumn{2}{|c|}{ ACENTO NUCLEAR } & \multicolumn{2}{c|}{$\%$} \\
\hline I & $\mathbf{V}$ & $\mathbf{I}$ & $\mathbf{V}$ & $\mathbf{I}$ & $\mathbf{V}$ \\
\hline & $\mathrm{L}+\mathrm{H}^{*} 30,4 \%$ & & & & $\mathrm{~L} \% 96,5 \%$ \\
$\mathbf{L}+\mathbf{H}^{*}$ & $\mathrm{~L}+>\mathrm{H}^{*} 28,5 \% 0^{31}$ & $\mathbf{L}^{*}$ & $\mathrm{~L} * 100 \%$ & $\mathbf{L} \%$ & $\mathrm{ML} \% 3,6 \%$ \\
\hline
\end{tabular}

Tabla 23

Por tanto, estas declarativas más largas se caracterizan por presentar un acento inicial invariablemente ascendente representando por el bitonal $/ \mathrm{L}+\mathrm{H}^{*} /$ y un acento nuclear bajo seguido de un tono bajo (/L*//L\%/). Véanse las invariantes en la tabla 24 , ilustradas en las figuras 18 y 19.

\begin{tabular}{|c|c|}
\hline \multicolumn{2}{|c|}{ Invariantes } \\
\hline ACENTO INICIAL & ACENTO NUCLEAR Y TONO DE FRONTERA \\
\hline $\mathrm{L}^{+} \mathrm{H}^{*}$ & $\mathrm{~L}^{*} \mathrm{~L} \%$ \\
\hline
\end{tabular}

Tabla 24

30 El $25 \%$ restante corresponde al etiquetaje $\left[\mathrm{L}^{*}+\mathrm{H}\right]$ realización con menor índice de aparición de $/ \mathrm{L} *+\mathrm{H} /$ que las variantes de $/ \mathrm{L}+\mathrm{H}^{*} /$.

31 El $41 \%$ restante corresponde al etiquetaje $\left[\mathrm{L}^{*}+\mathrm{H}\right](37,5 \%)$ y $\left[\mathrm{H}^{*}\right](3,5 \%)$ variantes de $/ \mathrm{L}^{*}+\mathrm{H} / \mathrm{y} / \mathrm{H}^{*} /$, respectivamente que tienen menor porcentaje de aparición que las variantes de $/ \mathrm{L}+\mathrm{H}^{*} /$. 


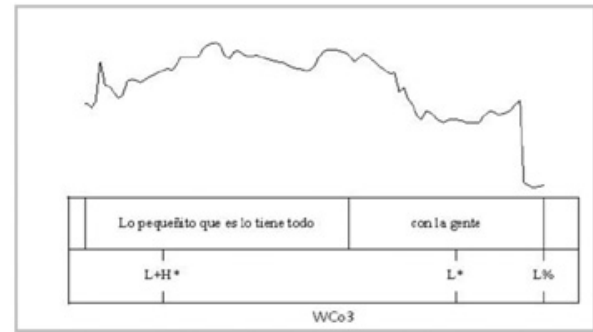

WCo3 (Sexo femenino)
Figuras 18-19

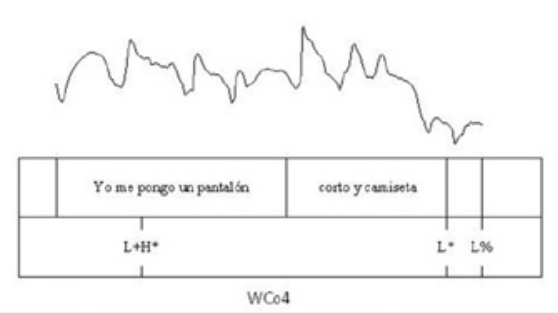

WCo4 (Sexo masculino)

\subsection{Invariantes: corpus experimental vs. espontáneo}

\subsubsection{Corpus experimental}

Según el análisis realizado, las oraciones SVO del corpus experimental se caracterizan por un patrón tonal inicial ascendente durante la vocal tónica $\left(/ \mathrm{L}+\mathrm{H}^{*} /\right)$, mientras que el último acento tiene un patrón descendente seguido de un tono de frontera bajo $(/ \mathrm{L} * \mathrm{~L} \% /)$. Como se puede observar en la figura 20, el hecho de que el sujeto presente expansión, no modifica el contorno del núcleo sintagmático que sigue siendo ascendente. En cambio, en las oraciones con expansión en el objeto, si bien el contorno final sigue siendo / $\mathrm{L}^{*} \mathrm{~L} \% /$, hay que tener en cuenta que este final corresponde a la expansión del sintagma y no a su núcleo que es $/ \mathrm{H}^{*} /$. Por tanto, la mayor extensión modifica la configuración del núcleo del sintagma final aunque no la del contorno tonal final de la oración.

\begin{tabular}{|c|c|c|c|c|}
\hline \multirow{2}{*}{ Tipo de oración } & \multicolumn{2}{|c|}{ SiNTAGMA NOMINAL } & \multicolumn{2}{|c|}{ SINTAGMA PREPOSICIONAL } \\
\hline & $\mathbf{N}$ & $\operatorname{Exp}$ & $\mathbf{N}$ & $\operatorname{Exp}$ \\
\hline \multicolumn{5}{|l|}{ Sin expansión } \\
\hline & & & $\mathbf{L} * \mathbf{L} \%$ & \\
\hline \multirow{2}{*}{$\begin{array}{l}\text { Con expansión } \\
\text { en sintagmas de } \\
\text { frontera }\end{array}$} & & & & \\
\hline & & $\mathbf{H}^{*}$ & $\mathbf{H}^{*}$ & $L * L \%$ \\
\hline
\end{tabular}

Figura 20

\subsubsection{Corpus espontáneo}

Hemos visto que con frecuencia se recoge en este corpus de contraste un acento inicial ascendente durante la tónica $\left(/ \mathrm{L}+\mathrm{H}^{*} /\right)$, aunque si las oraciones tienen un solo pico tonal este esquema alterna con el ascenso después del acento $\left(/ \mathrm{L}^{*}+\mathrm{H} /\right)$. El final de las oraciones, en cambio, 
se caracteriza siempre por un tono de frontera bajo $(/ \mathrm{L} \% /)$ y la altura del contorno del acento nuclear es, asimismo, bajo (/L*/). Véanse las invariantes y sus ilustraciones en la figura 21 .

\begin{tabular}{|c|c|c|}
\hline Tipo de oración & Acento inicial & Acento nuclear y tono de frontera \\
\hline \multirow{2}{*}{$-1 P M x$} & $\mathbf{L} *+\mathbf{H}$ & \\
\hline & $\mathbf{L}+\mathbf{H}^{*}$ & \multirow[t]{3}{*}{$\mathbf{L} * \mathbf{L} \%$} \\
\hline \multirow[t]{2}{*}{$+1 P M x$} & & \\
\hline & $\mathbf{L}+\mathbf{H}^{*}$ & \\
\hline
\end{tabular}

Figura 21

\subsection{Invariantes $y$ variantes}

El análisis realizado permite completar la propuesta tonal esbozada en Dorta (ed. 2013). Véanse, en primer lugar, los acentos tonales en la figura 22.

\begin{tabular}{|c|c|c|}
\hline \multicolumn{3}{|c|}{ ACENTOS TONALES } \\
\hline \multicolumn{2}{|c|}{ ESTRUCTURA PROFUNDA } & JRA SUPERFICIAL \\
\hline \multicolumn{2}{|c|}{ Invariante fonológica } & Variante \\
\hline$/ \mathrm{L} *+\mathrm{H} /$ & & {$\left[\mathrm{L}^{*}+\mathrm{H}\right]$} \\
\hline$/ \mathrm{L}+\mathrm{H}^{*} /$ & & $\begin{array}{c}{\left[\mathrm{L}+\mathrm{H}^{*}\right]} \\
{\left[\mathrm{L}+>\mathrm{H}^{*}\right]}\end{array}$ \\
\hline$/ \mathrm{H}^{*} /$ & & $\begin{array}{c}{\left[\mathrm{H}^{*}\right]} \\
{\left[\mathrm{iH}^{*}\right]} \\
{\left[\mathrm{L}+\mathrm{H}^{*}\right]} \\
{\left[\mathrm{L}+>\mathrm{H}^{*}\right]} \\
{\left[\mathrm{L}+! \mathrm{H}^{*}\right]} \\
{\left[\mathrm{L}+>{ }_{i} \mathrm{H}^{*}\right]}\end{array}$ \\
\hline$/ \mathrm{L} * /$ & & $\begin{array}{c}{\left[\mathrm{L}^{*}\right]} \\
{\left[\mathrm{H}+\mathrm{L}^{*}\right]}\end{array}$ \\
\hline
\end{tabular}

Figura 22. Representación de la estructura profunda y superficial de los acentos tonales 
$\left.1^{\circ}\right)$ Las invariantes bitonales $/ \mathrm{L}+\mathrm{H} / \mathrm{y} / \mathrm{L}+\mathrm{H}^{*} /$ solo aparecen en el acento inicial: $/ \mathrm{L}+\mathrm{H}^{*} /$ es la invariante más general en los dos tipos de corpus ( $C E$ y $C S)$, pero en el espontáneo interactúa con $/ \mathrm{L}^{*}+\mathrm{H} / \mathrm{si}$ las oraciones son breves.

$2^{\circ}$ ) Cuando se trata de la expansión del sintagma sujeto o del núcleo del sintagma objeto aparecen las variantes del acento monotonal alto $/ \mathrm{H}^{*} /$ anotadas en Dorta (ed. 2013), esto es, $\left[\mathrm{H}^{*}\right],\left[\mathrm{iH}^{*}\right]$ y $\left[\mathrm{L}+\mathrm{H}^{*}\right]$-para describir otro tipo de posiciones ${ }^{32}$ - junto a $\left[\mathrm{L}+>\mathrm{H}^{*}\right] ;\left[\mathrm{L}+\mathrm{H}^{*}\right]$ y $\left[\mathrm{L}+>{ }_{i} \mathrm{H}^{*}\right]$.

$\left.3^{\circ}\right)$ Por último, el acento monotonal $/ \mathrm{L}^{*} /$ presenta dos variantes: [L*]: aparece en el sintagma verbal de las oraciones sin expansión del corpus experimental y en el núcleo final de prácticamente todas las oraciones, mientras que la existencia de $\left[\mathrm{H}+\mathrm{L}^{*}\right]$ se reduce a las oraciones espontáneas más breves, esto es, la de un una cumbre tonal.

En relación con los tonos de frontera (figura 23), tal como sucedía en Dorta (ed. 2013), $/ \mathrm{L} \% /$ marca la trayectoria descendente en el final de las declarativas con dos variantes: [L\%], que aparece en los dos tipos de corpus y [ML\%], que solo se registró en las oraciones más espontáneas.

\begin{tabular}{|c|c|c|}
\hline \multicolumn{3}{|c|}{ TONOS DE FRONTERA } \\
\hline \multicolumn{2}{|c|}{ ESTRUCTURA PROFUNDA } & ESTRUCTURA SUPERFICIAL \\
\hline \multicolumn{2}{|c|}{ Invariante fonológica } & Variante \\
\hline$/ \mathrm{L} \% /$ & {$\left[\begin{array}{c|c}\mathrm{[L} \%] \\
{[\mathrm{ML} \%]}\end{array}\right.$} \\
\hline
\end{tabular}

Figura 23. Representación de la estructura profunda y superficial de los tonos de frontera

En Dorta (ed. 2013) la variante [ML\%] solo se registró puntualmente en la modalidad declarativa (agudos de Caracas) y en las interrogativas circunflejas agudas de las zonas venezolanas (Caracas y Bolívar).

\section{Conclusiones y discusión de los resultados}

En este trabajo hemos intentado sistematizar la variabilidad tonal de las declarativas en el marco del Sp_ToBI para poder extraer las invariantes más frecuentes. Destacamos aquí el comportamiento tonal en el inicio y en el final de las curvas.

$\left.\mathbf{1}^{\mathbf{0}}\right)$ En relación con el primer acento tonal, hemos encontrado como tendencia más general $/ \mathrm{L}+\mathrm{H}^{*} /$ (estructura profunda o invariante), tanto en el corpus experimental como en el de contraste, si bien en este último corpus dicho acento alterna con el esquema fonológico $/ \mathrm{L}^{*}+\mathrm{H} /$ en las oraciones monocumbres. Por tanto, los resultados han evidenciado que si bien la secuencia LH se actualiza generalmente dentro de los límites de la tónica, con ascenso

32 En Dorta ed. 2013 la invariante $/ \mathrm{H}^{*} /$ se localizaba en el primer acento agudo de Santiago de Cuba y en el segundo de todas las variedades $\left(\left[\mathrm{H}^{*}\right]\right)$; también aparece en el acento nuclear de las interrogativas sin expansión: $\left[\mathrm{H}^{*}\right] ;\left[{ }_{i} \mathrm{H}^{*}\right]$ o $\left[\mathrm{L}+\mathrm{H}^{*}\right]$. Hay que tener en cuenta que en este libro solo se analizan oraciones del tipo SVO y no las de expansión en los sintagmas de frontera como se ha hecho en el presente trabajo. 
posterior $\left(\left[\mathrm{L}+>\mathrm{H}^{*}\right]\right)$ o sin él $\left(\left[\mathrm{L}+\mathrm{H}^{*}\right]\right)$ (estructuras superficiales o variantes), lo relevante es que, salvo la excepción anotada, el crecimiento del ascenso tonal incide en la vocal acentuada: $/ \mathrm{L}+\mathrm{H}^{*} /$.

Por tanto, nuestros resultados coinciden generalmente con los de Dorta ed., 2013; Díaz et al. en prensa; Dorta, Díaz y Hernández en prensa o Dorta, Martín y Díaz, 2015 en los que se aborda el etiquetaje prosódico del español de la zona urbana de Canarias, Cuba, Venezuela y Colombia en estilo formal y/o espontáneo. En efecto, como en los citados trabajos, consideramos que la invariante tonal del primer acento para declarativas es frecuentemente $/ \mathrm{L}+\mathrm{H}^{*} /$ y no su variante $\left[\mathrm{L}+>\mathrm{H}^{*}\right]$, tal como apuntan algunos autores para las declarativas de los dialectos peninsulares (Prieto y Roseano eds., 2010: Estebas y Prieto, capítulo 2 y López-Bobo y Cuevas Alonso, capítulo 3), de los hispanoamericanos, como la zona andina de Venezuela o Ecuador (Mérida y Quito. Prieto y Roseano eds., 2010: Astruc et al., capítulo 7 y O'Rourke, capítulo 8), Santiago de los Caballeros (Santo Domingo. Prieto y Roseano eds., 2010: Willis, capítulo 5) y México D.F. (Prieto y Roseano eds., 2010: de la Mota et al., capítulo 11) o de la variedad canaria del español de Gran Canaria (Prieto y Roseano eds., 2010: Cabrera y Vizcaíno capítulo 4).

$\left.2^{\mathbf{0}}\right)$ El acento nuclear y el tono de frontera final se caracterizan siempre en el corpus experimental como $/ \mathrm{L} * / / \mathrm{L} \% /$. En el corpus de contraste sucede lo mismo, aunque $/ \mathrm{L} * /$ puede actualizarse en las oraciones que constan de un único acento con la variante $\left[\mathrm{H}+\mathrm{L}^{*}\right]$ y no [L*].

Por tanto, las declarativas de La Gomera presentan por lo general en ambos corpus un tono bajo durante la tónica y un tono de frontera bajo (/L* $\mathrm{L} \% /)$, tal como refleja la bibliografía más reciente correspondiente al español de Canarias (Prieto y Roseano eds., 2010: Cabrera y Vizcaíno, capítulo 4; Dorta ed., 2013). Este esquema descendente es el más frecuente en el español general (Sosa, 1999; Prieto y Roseano eds., 2010: Estebas y Prieto, capítulo 2 y López-Bobo y Cuevas-Alonso, capítulo 3) y en la mayor parte de dialectos hispanoamericanos (Sosa, 1999; Dorta ed., 2013; Díaz et al., en prensa; Dorta, Díaz y Hernández, en prensa) en los que, incluso cuando se da un contorno circunflejo final, esto es, $/ \mathrm{H}^{*} \mathrm{~L} \% / \mathrm{o} / \mathrm{L}+\mathrm{H}^{*} \mathrm{~L} \% /$, este no impide la trayectoria descendente del contorno final. $\mathrm{Al}$ respecto, Sosa llega a la conclusión de que la configuración $\mathrm{H}^{*} \mathrm{o} \mathrm{L}+\mathrm{H}^{*}$ en el último segmento tónico solo tiene cierta relevancia en Ciudad de México (1999: 196-197).

\section{Bibliografía}

Beckman, M.; M. Díaz Campos; J. T. McGory y A. M. Terrell (2002): "Intonation across Spanish in the Tones and Break Indices framework", Probus 14, págs. 9-36.

Coseriu, E. (1962): Teoría del lenguaje y lingüistica general, Madrid, Gredos.

Díaz, Ch. (2013): Contribución al Atlas Prosódico de Canarias (AMPERCan): Declarativas e interrogativas de La Gomera (Islas Canarias), Tesis doctoral, Santa Cruz de Tenerife, Universidad de La Laguna.

Díaz, CH. y J. Dorta (en prensa): “¿Coexistencia de configuraciones tonales en la variedad isleña de La Gomera?».

Díaz, CH.; J. Dorta; E. Mora y M. Muñetón (en prensa): «Intonation in border areas of the northern Andes: Mérida (Venezuela) and Medellin (Colombia)». Issues in Hispanic and Lusophone Linguistics. John Benjamins. 
Dorta, J. (2000): “Entonación Hispánica: No pronominales vs. Pronominales", Lingüística Española Actual, Madrid, Arco/ Libros, págs. 51-76.

Dorta, J. (2013): «Estudio fonético-fonológico de la entonación declarativa e interrogativa canaria en voz femenina». En A. Pamies (ed.): De lingüística, traducción y léxico-fraseología. Homenaje a Juan de Dios Luque Durán. Interlingüística 111, págs. 173 - 197.

Dorta, J. (ed. 2013): Estudio comparativo preliminar de la entonación de Canarias, Cuba y Venezuela. La Página ediciones S. L. /Colección Universidad, Madrid-Tenerife. Participan: Josefa Dorta, Elsa Mora, Beatriz Hernández Díaz, Chaxiraxi Díaz Cabrera, Nelson Rojas, José Antonio Martín Gómez y Carolina Jorge.

Dorta, J. y CH. Díaz (2014): "Reconocimiento perceptivo de patrones interrogativos coexistentes en Canarias", Fortunatae, Homenaje al Dr. Fremiot Hernández González, págs. 115-127.

Dorta, J., CH. Díaz y B. Hernández (2015): “La entonación cubana en zonas rurales: La Habana y Santa Clara”. En A. Cabedo Nebot (ed.): Perspectivas actuales en el análisis fónico del habla: tradición y avances en la fonética experimental, anejo $\mathrm{n}^{\mathrm{o}} 7$ de la revista Normas. Revista de Estudios Lingüísticos Hispánicos, págs. 45-55.

Dorta, J., J. A. Martín Gómez y CH. Díaz (2015): “Continuidad prosódica en habla experimental y espontánea de Canarias y Cuba: variación y rango tonal en las interrogativas no pronominales". En Kirsten Jeppesen Kragh, Jan Lindschouw (éds.): Les variations diasystématiques et leurs interdépendances dans les langues romanes. Travaux de Linguistique Romane. Sociolinguistique, dialectologie, variation. Collection dirigée par Emili Casanova (València), Jean-Paul Chauveau (Nancy, ATILF), Hans Goebl (Salzbourg). Ouvrage publié avec l'appui de l'Académie Royale des Sciences et Belleslettres de Danemark et du Lektor Knud Henders Legatfond. Strasbourg, págs. 145-159.

Estebas Vilaplana, E. y P. Prieto (2008): “La notación prosódica del español: una revisión del Sp_ToBI", Estudios de Fonética Experimental, 17, Barcelona, págs. 265-283.

Face, T. y P. Prieto (2007): "Rising accents in Castilian Spanish: a revision of Sp-ToBI". En G. Elordieta y M. Vigário (eds.): Journal of Portuguese Linguistics (special issue on Prosody of Iberian Languages), 6.1, págs. 117-146.

Fernández Planas, A. M. y E. Martínez Celdrán (2003): “El tono fundamental y la duración: dos aspectos de la taxonomía prosódica en dos modalidades de habla (enunciativa e interrogativa) del español", Estudios de Fonética Experimental, 12, Barcelona, págs. 165-200.

Hernández, B; CH. Díaz y C. Jorge (2014): «Declarativas e interrogativas en zonas rurales de Canarias». En Y. Congosto Martín, M. L. Montero Curiel y A. Salvador Plans (eds.): Fonética Experimental, Educación Superior e Investigación. T. III. Prosodia. Madrid, Editorial Arco Libros, S.L., págs. 245-265.

Hernández Díaz, B.; J. Dorta y CH. Díaz (2011): “La entonación de las declarativas simples en voz femenina. Zonas urbanas de las islas canarias". En A. Hidalgo, Y. Congosto y M. Quilis (eds.): El estudio de la prosodia en España en el S. XXI. Perspectivas y Ámbitos; Anejos de Quaderns de Filologia de la Universitat de València, págs. 109-124.

Hualde, J. I. (2003): “El modelo métrico y autosegmental”. En P. Prieto (ed.): Teorías de la entonación, Barcelona, Ariel, págs. 155-184.

López Bobo, M. J.; C. Muñiz Cachón; L. Díaz Gómez; N. Corral Blanco; D. Brezmes Alonso y M. Alvarellos Pedrero (2007): "Análisis y representación de la entonación. Replanteamiento metodológico en el marco del proyecto AMPER”. En J. Dorta (ed.): 
La prosodia en el ámbito lingüistico románico, Santa Cruz de Tenerife: La Página Ediciones, S.L. (Colección Universidad), págs. 17-34.

Pamies-Bertrán, A; A.M. Fernández-Planas; E. Martínez-Celdrán; A. Ortega-Escandell y M. C. Amorós (2002): “Umbrales tonales en español peninsular”. En J. Díaz García (ed.): Actas del II Congreso de Fonética Experimental, Sevilla, Universidad de Sevilla, págs. 272-278.

Pierrehumbert, J. (1980): The phonetics and phonology of English intonation, tesis doctoral, MIT.

Prieto, P. y P. Roseano (eds. 2010): Transcription of Intonation of the Spanish Language, Lincom Europa: München.

Romano, A; J.P. Lai y S. Roullet (2005): “La méthodologie AMPER”, Géolinguistique Hors Serie 3, págs. 1-5.

Rietveld, A. y C. Gussenhoven (1985): "On the relation between pitch excursion size and prominence", Journal of Phonetics, New York, Academic Press, 13, págs. 299-308.

Sosa, J.M. (1999): La entonación del español, su estructura fónica, variabilidad y dialectología, Madrid, Cátedra.

Toledo, G. (2006): “Tonos estrellados: una argumentación”, Estudios de Fonética Experimental, 15, Barcelona, págs. 101-131.

Torres Álvarez, M.I. (2000): “Oraciones cortas vs. Oraciones largas: diferencias en el patrón entonativo", Revista de Filología de la Universidad de La Laguna, 18, págs. 383-397.

http://prosodia.upf.edu/sp_tobi/en/ (20-06-2013)

http://www.fon.hum.uva.nl/praat/ (03-03-2012)

http://prosodia.upf.edu/atlasentonacion/cerca/index.php (20-06-2013) 